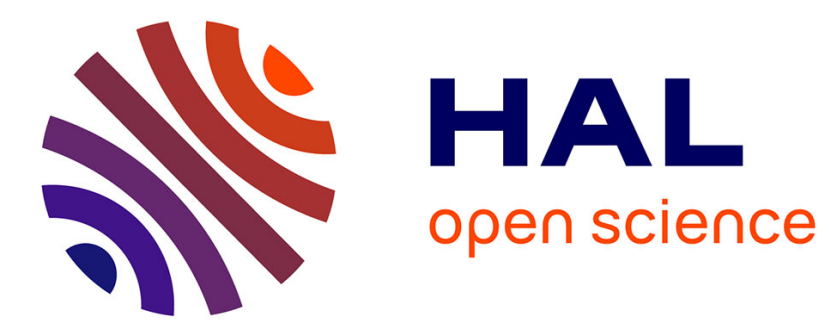

\title{
Theoretical analysis of the zigzag instability of a vertical columnar vortex pair in a strongly stratified fluid Paul Billant, Jean-Marc Chomaz
}

\section{To cite this version:}

Paul Billant, Jean-Marc Chomaz. Theoretical analysis of the zigzag instability of a vertical columnar vortex pair in a strongly stratified fluid. Journal of Fluid Mechanics, 2000, 419 (september), pp.29-63. 10.1017/S0022112000001166 . hal-01025369

HAL Id: hal-01025369

https://hal-polytechnique.archives-ouvertes.fr/hal-01025369

Submitted on 11 Sep 2014

HAL is a multi-disciplinary open access archive for the deposit and dissemination of scientific research documents, whether they are published or not. The documents may come from teaching and research institutions in France or abroad, or from public or private research centers.
L'archive ouverte pluridisciplinaire HAL, est destinée au dépôt et à la diffusion de documents scientifiques de niveau recherche, publiés ou non, émanant des établissements d'enseignement et de recherche français ou étrangers, des laboratoires publics ou privés. 


\title{
Theoretical analysis of the zigzag instability of a vertical columnar vortex pair in a strongly stratified fluid
}

\author{
By PAUL BILLANT T, ${ }^{1, \dagger}$ AND JEAN-MARC CHOMAZ ${ }^{1}$ \\ ${ }^{1}$ LadHyX, CNRS, École Polytechnique, F-91128 Palaiseau Cedex, France \\ ${ }^{2}$ Météo-France CNRM Toulouse, 42 avenue Coriolis, F-31057 Toulouse, France
}

(Received 1 November 1999)

A general theoretical account is proposed for the zigzag instability of a vertical columnar vortex pair recently discovered in a strongly stratified experiment.

The linear inviscid stability of the Lamb-Chaplygin vortex pair is analysed by a multiple-scale expansion analysis for small horizontal Froude number $\left(F_{h}=U / L_{h} N\right.$, where $U$ is the magnitude of the horizontal velocity, $L_{h}$ the horizontal lengthscale and $N$ the Brunt-Väisälä frequency) and small vertical Froude number $\left(F_{v}=U / L_{v} N\right.$, where $L_{v}$ is the vertical lengthscale) using the scaling of the equations of motion introduced by Riley, Metcalfe \& Weissman (1981). In the limit $F_{v}=0$, these equations reduce to two-dimensional Euler equations for the horizontal velocity with undetermined vertical dependence. Thus, at leading order, neutral modes of the flow are associated, among others, to translational and rotational invariances in each horizontal plane. To each broken invariance is related a phase variable that may vary freely along the vertical. Conservation of mass and potential vorticity impose at higher order the evolution equations governing the phase variables that we derive for $F_{h} \ll 1$ and $F_{v} \ll 1$ in the spirit of phase dynamics techniques established for periodic patterns. In agreement with the experimental observations, this asymptotic analysis shows the existence of an instability consisting of a vertically modulated rotation and a translation of the columnar vortex pair perpendicular to the travelling direction. The dispersion relation as well as the spatial eigenmode of the zigzag instability are determined. The analysis predicts that the most amplified vertical wavelength should scale as $U / N$ and the maximum growth rate as $U / L_{h}$.

Our main finding is thus that the typical thickness of the ensuing layers will be such that $F_{v}=O(1)$ and not $F_{v} \ll 1$ as assumed by Riley et al. (1981) and Lilly (1983). This implies that such strongly stratified flows are not described by twodimensional horizontal equations. These results may help to understand the layering commonly observed in stratified turbulence and the fundamental differences with strictly two-dimensional turbulence.

\section{Introduction}

A striking and prominent feature of strongly stratified turbulence observed in experiments (Lin \& Pao 1979; Browand, Guyomar \& Yoon 1987; Hopfinger 1987; Lin et al. 1992; Chomaz et al. 1993; Flór \& Heijst 1996; Fincham, Maxworthy \&

$\dagger$ Present address: Météo-France CNRM Toulouse, 42 avenue Coriolis, F-31057 Toulouse, France. 
Spedding 1996; Spedding, Browand \& Fincham, 1996; Spedding 1997; Bonnier, Eiff $\&$ Bonneton 2000) and numerical simulations (Riley, Metcalfe \& Weissman 1981; Métais \& Herring 1989; Herring \& Métais 1989; Staquet \& Riley 1989; Kimura \& Herring 1996) is the emergence of coherent horizontal pancake vortices organized in decoupled layers. Layers and turbulent patches are also widely observed in the oceans (Gregg 1987) and atmosphere. As reflected by the term 'pancake', these vortices are highly anisotropic with predominantly horizontal motions. The dissipation as well as the vertical fluxes of energy and mass of stratified turbulent flows depend crucially on the dynamical properties of this 'lasagne' structure. Up to now, no theory has been able to describe this layering process and predict the layer thickness.

Layering may be observed after three-dimensional turbulence has collapsed owing to the gravitational restoring force (Stillinger, Helland \& Van Atta 1983; Itsweire, Helland \& Van Atta 1986; Browand et al. 1987; Métais \& Herring 1989). Layers may also emerge from an initially vertically coherent flow as shown by Herring \& Métais (1989) in numerical simulations of forced stratified turbulence. With the purpose of investigating the spectral dynamic of forced stratified turbulence, a turbulent flow has been forced only two-dimensionally. Initially, this flow is vertically uniform, i.e. purely two-dimensional. Interestingly, a three-dimensional perturbation subsequently added has been observed to grow exponentially, leading to the formation of decoupled layers. An intriguing feature that remains unexplained is that the growth rate of this instability is independent of the Brunt-Väisälä frequency.

Fincham et al. (1996) (see also Spedding et al. 1996) have experimentally observed decoupled layers behind a rake of vertical bars. During the time evolution, the shed vortices quickly decorrelate and several decoupled layers of quasi-two-dimensional vortices are produced. The resulting vertical shear is observed to account for $90 \%$ of the dissipation of kinetic energy.

In the different context of quasi-geostrophic flows, i.e. strongly stratified and rapidly rotating flows, Dritschel \& Torre Juárez (1996) have reported in numerical experiments that an instability is also responsible for the vertical decoupling of initially columnar multi-vortex flows. This instability eventually produces vortices with a height-to-width ratio about $f / 3 N$, where $f$ is the background vorticity and $N$ the Brunt-Väisälä frequency.

In order to characterize the physical mechanism for the layering, we have investigated the dynamic of a columnar vertical vortex pair in a stratified fluid (non-rotating, i.e. $f=0$ ). This experimental study (Williamson \& Chomaz 1997; Billant \& Chomaz $2000 a$ ) confirms that the layering may arise from an instability when the fluid is strongly stratified. Along the vertical, the columnar vortex pair, initially uniform, is sinusoidally twisted and bent perpendicularly to the travelling direction. Such an instability, called the zigzag instability, generates no appreciable change in the dipole structure in any horizontal cross-section; the dipole appears only to be translated and turned as a solid body. Ultimately, the columnar vortex pair is literally chopped into thin independent 'pancake' dipoles.

All these observations support the idea that the layering may arise from an instability. As a first step toward an understanding of this layering phenomenon, it is instructive to consider the scaling analysis proposed by Riley et al. (1981) and Lilly (1983) to describe strongly stratified flows. When inertial forces are small in comparison with buoyancy, i.e. when the horizontal Froude number is small $F_{h}=U / L_{h} N \ll 1$, where $U$ is the magnitude of the horizontal velocity and $L_{h}$ horizontal lengthscale, and when the vertical scale $L_{v}$ is large in comparison with the buoyancy length $U / N$, i.e. when the vertical Froude number is also small $F_{v} \ll 1$, the 
scaled equations for the horizontal velocity reduce to two-dimensional incompressible Euler equations with undetermined vertical dependence while the vertical velocity goes to zero.

A physical interpretation of the results of this scaling analysis may be given. In contrast with homogeneous flows where vorticity fluxes through material surfaces are conserved and vorticity tubes are simply advected by virtues of Kelvin and Helmholtz theorems respectively, the vorticity in stratified flows is not conserved since the baroclinic torque may create vorticity. The only invariants we are left with in the Boussinesq approximation are the mass, the density and the scalar quantity $\Pi=\omega \cdot \nabla \rho$ (where $\omega$ is the vorticity and $\rho$ the density) known as Ertel's potential vorticity. When the flow is strongly stratified, the deformations of the isopycnal surfaces are of the order $F_{v} U / N$. Therefore, if we assume as Riley et al. (1981) and Lilly (1983) that $L_{v} \gg U / N$, then the isopycnal surfaces are flat at leading order. The Ertel theorem thus implies the conservation of vertical vorticity and mass conservation imposes that the horizontal velocity be non-divergent in the horizontal plane. The dynamic in each layer is therefore governed by the incompressible two-dimensional Euler equations.

Therefore, the analysis of Riley et al. (1981) and Lilly (1983) accounts for the observed quasi-two-dimensional motion, each superposed two-dimensional horizontal flow being free to evolve independently of the others. However, at leading order, there is no tendency for a stack of horizontal flows which are initially vertically coherent to decouple rather than to remain vertically rigid. As shown by Majda \& Grote (1998), vertical decoupling occurs if a mean horizontal flow varying along the vertical direction is added to a vertically coherent flow. It is important to note that the magnitude and the vertical shear of this mean horizontal flow can be chosen arbitrarily and are externally imposed in the Majda \& Grote (1998) study. However, in the vortex pair experiments, the zigzag instability develops in the absence of a vertically sheared mean horizontal flow so that the analysis of Majda \& Grote (1998) is not adequate to explain this spontaneous layering.

Since horizontal layers of two-dimensional flow evolve independently in the zero Froude number limit $F_{v}=0$, the origin of the spontaneous layering must be sought in non-zero Froude-number effects. This is the aim of the present paper in the case of the columnar vortex pair in an attempt to explain the zigzag instability described above. Although applied to the vortex pair case, our approach is general and could be applied to any steady horizontal flow which is initially vertically uniform in a strongly stratified fluid. The stability problem is solved asymptotically for small Froude numbers using the scaling of Riley et al. (1981) for strongly stratified flows. At leading order, the zero Froude number limit is recovered and the perturbations are governed in each horizontal plane by the linearized two-dimensional Euler equations. Because nothing couples the two-dimensional horizontal flows together, infinitely small horizontal translation and rotation of the basic flow in any horizontal plane are neutral perturbations at leading order. Although there are several neutral modes associated to each of the invariances of the equations of motion, we focus in the present case of a columnar vortex pair on the neutral perturbation deriving from the translational invariance in the direction perpendicular to the dipole travelling direction, i.e. in the $y$-direction (figure 1). To obtain the mathematical structure of this neutral mode, let us denote by $\psi_{0}(x, y)$ the basic state streamfunction of a dipole expressed in the co-moving frame of reference (figure 1). The translational invariance in the $y$-direction implies that $\psi_{0}(x, y-\eta)$, where $\eta$ is a constant, is an exact solution of the nonlinear Euler equations. Physically, $\eta$ corresponds to the $y$ location of the 


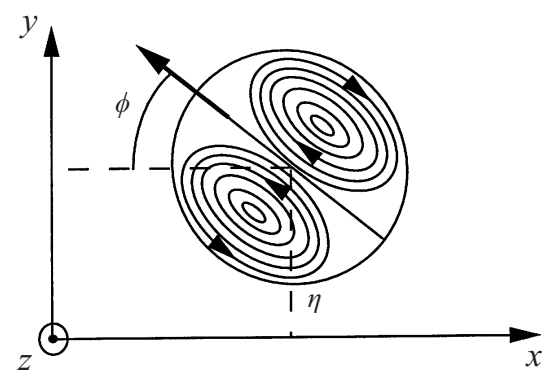

FiguRE 1. Definition of the relevant phase parameters involved in the instability: the $y$ position $\eta$ and the angle of propagation $\phi$ of the dipole. The unperturbed dipole initially propagates along the $x$-axis with $\eta=0$ and $\phi=0$.

dipole centre (figure 1). The vertical decoupling in the limit $F_{v}=0$ means further that $\eta$ can be chosen arbitrarily in each layer and therefore may have any vertical dependence, $\eta \equiv \eta(z)$. For an infinitesimal translation $\eta(z) \ll 1$, the streamfunction can be Taylor expanded, keeping only the first order

$$
\psi_{0}(x, y-\eta(z))=\psi_{0}(x, y)-\eta(z) \frac{\partial \psi_{0}}{\partial y} .
$$

Interpreted in the linear stability framework, the first term is the basic state whereas the second term is an infinitesimal perturbation consisting of a small translation perpendicular to the travelling direction which is neutral since (1.1) is a solution of the full nonlinear equations in the limit $F_{v}=0$. Therefore, $\tilde{\psi}(x, y, z)=\eta(z) \partial \psi_{0} / \partial y$ is the neutral 'phase' mode associated with the translation invariance in $y$.

In order to determine the effect of small Froude number on the stability of the stack of horizontal sheets with respect to a small $y$ translation perturbation, we insert the neutral perturbation $\tilde{\psi}=\eta \partial \psi_{0} / \partial y$ for zero Froude numbers into the full linearized inviscid equations. As we solve successively the iteration equations, solvability conditions yield evolution equations over slow timescales for the phase variables governing the neutral modes.

This multiple-scale analysis is similar to the phase techniques widely used to study the stability of cellular flows which arises in Rayleigh-Bénard convection or CouetteTaylor flows and to describe pattern dynamics in time periodic chemical reactions (Pomeau \& Manneville 1979; Kuramoto 1984; Fauve, Bolton \& Brachet 1987; Fauve 1987; Manneville 1990). All the observed long-wavelength instabilities of convective rolls have been recovered with the phase dynamics method (Pomeau \& Manneville 1979; Cross 1983; Cross \& Newell 1984; Fauve et al. 1987). The Eckhaus and zigzag instabilities are well-known examples.

For the present strongly stratified columnar vertical vortex pair, this approach leads to two coupled linear evolution equations for the $y$ position of the dipole $\eta(z, t)$ and the angle of propagation $\phi(z, t)$ (see figure 1) which read in non-dimensional form

$$
\begin{gathered}
\frac{\partial \eta}{\partial t}=\phi, \\
\frac{\partial \phi}{\partial t}=\left(D+F_{h}^{2} g_{1}\right) F_{v}^{2} \frac{\partial^{2} \eta}{\partial z^{2}}+g_{2} F_{v}^{4} \frac{\partial^{4} \eta}{\partial z^{4}},
\end{gathered}
$$

up to fourth order in $F_{v}$ and $\left(F_{v} F_{h}\right)^{1 / 2}$. The characteristic velocity and horizontal lengthscale are chosen as the propagating velocity and radius $R$ of the dipole. 
The vertical coordinate $z$ is non-dimensionalized by $L_{v}$ so that the vertical Froude number $F_{v}$ measures the magnitude of the vertical wavenumber. The only way in which this multiple-scale analysis differs from classical phase techniques is that disturbances are assumed to have a long wavelength compared to the buoyancy length $U / N$ whereas usually, the disturbance wavelength is assumed to be long relative to the characteristic horizontal scale $L_{h}$ of the pattern. The coefficients appearing in (1.3), $D=-3.67, g_{1}=-56.4, g_{2}=-16.1$, are computed exactly from the solvability conditions using the Lamb-Chaplygin solution (Lamb 1932; Batchelor 1967; Meleshko \& van Heijst 1994) as a dipole model.

Most interestingly, we shall see that equations (1.2)-(1.3) describe an instability with a behaviour closely resembling the zigzag instability observed experimentally (Billant \& Chomaz 2000a).

The paper is organized as follows. The general stability problem is formulated in $\S 2$. The governing equations are recalled in $\S 2.1$ and then non-dimensionalized in $\S 2.2$ following the scaling of Riley et al. (1981). The Lamb-Chaplygin dipole is presented in $\S 2.3$ and the full linearized stability equations are outlined in $\S 2.4$. In $\S 3$, the multiple-scale analysis leading to (1.2)-(1.3) is carried out. This asymptotic analysis provides also a clear physical explanation for the instability mechanism. The stability of (1.2)-(1.3) is next analysed in $\S 4$.

\section{Stability problem}

\subsection{Governing equations}

The flow is assumed to satisfy the Boussinesq approximation for the Euler equation

$$
\frac{\mathrm{D} \boldsymbol{u}}{\mathrm{D} t}=-\frac{1}{\rho_{0}} \nabla P-g \frac{\rho^{\prime}}{\rho_{0}} \boldsymbol{e}_{z},
$$

together with the incompressibility condition

$$
\nabla \cdot \boldsymbol{u}=0
$$

leading to

$$
\frac{\mathrm{D} \rho}{\mathrm{D} t}=0 .
$$

where $\boldsymbol{u}=\left(u_{x}, u_{y}, u_{z}\right)$ is the velocity vector in Cartesian coordinates $(x, y, z)$, with $z$ along the vertical direction, $P$ the pressure, $g$ the gravity, $\boldsymbol{e}_{z}$ the unit vector pointing upward and the density

$$
\rho(\boldsymbol{x}, t)=\rho_{0}+\bar{\rho}(z)+\rho^{\prime}(\boldsymbol{x}, t),
$$

is expressed as the sum of a constant reference density $\rho_{0}$, a linear mean density profile $\bar{\rho}(z)$ and a perturbation density $\rho^{\prime}(\boldsymbol{x}, t)$. Viscous and mass diffusion could be taken into account in the analysis although the algebra would be more cumbersome. An important quantity conserved by $(2.1 a-c)$ following the motion is Ertel's potential vorticity $\Pi=\nabla \times \boldsymbol{u} \cdot \nabla \rho$. In what follows, we use either Cartesian coordinates or cylindrical coordinates $(r, \theta, z)$ with $x=r \cos \theta$ and $y=r \sin \theta$.

\subsection{Scaling analysis}

We begin by non-dimensionalizing the equations when buoyancy effects are dominant following the well-known scaling analysis used by Riley et al. (1981). We denote by $U$ a characteristic horizontal velocity scale, $L_{h}$ a horizontal lengthscale ( $L_{h}$ will later be the dipole radius $R$ ) and $L_{v}$ a vertical lengthscale ( $L_{v}$ will be taken to be the 
order of magnitude of the vertical wavelength of the disturbances). The timescales $L_{h} / U$ and $1 / N$ characterize horizontal advective motions and internal gravity waves, respectively, where $N=\sqrt{-\left(g / \rho_{0}\right) \partial \bar{\rho} / \partial z}$ is the Brunt-Väisälä frequency. The ratio of these two time scales defines a horizontal Froude number

$$
F_{h}=\frac{U}{L_{h} N},
$$

which measures the effect of inertial forces in comparison with buoyancy force. Since the basic state consists of two-dimensional vortices, we choose $T=L_{h} / U$ as timescale. When $F_{h}$ is small, this effectively filters out fast internal waves from slow horizontal advective motions. The pressure scale $\rho_{0} U^{2}$ is deduced from the horizontal momentum equation. The density equation imposes that the magnitudes of the vertical velocity $W$ and density perturbations $R$ are related by $W=R\left(g F_{h} / \rho_{0} N\right)$. Inserted in the vertical momentum equation, this scale for the vertical velocity indicates that when $F_{h} \ll 1$, the vertical acceleration term is negligible compared to the buoyancy term. Thus, the only way to balance the vertical pressure gradient is by density perturbations giving $R=\rho_{0} U^{2} /\left(g L_{v}\right)$ and $W=F_{h} F_{v} U$, where the vertical Froude number

$$
F_{v}=\frac{U}{L_{v} N},
$$

compares the vertical scale $L_{v}$ to the buoyancy length $L_{b}=U / N$. The length $L_{b}$ can be interpreted as the vertical displacement of a fluid parcel if all its kinetic energy were converted to potential energy.

Keeping the same notation for the dimensionless variables, the non-dimensional equations are

$$
\begin{aligned}
\frac{\partial \boldsymbol{u}_{h}}{\partial t}+\boldsymbol{u}_{h} \cdot \nabla_{h} \boldsymbol{u}_{h}+F_{v}^{2} u_{z} \frac{\partial \boldsymbol{u}_{h}}{\partial z} & =-\nabla_{h} P, \\
F_{h}^{2}\left(\frac{\partial u_{z}}{\partial t}+\boldsymbol{u}_{h} \cdot \nabla_{h} u_{z}+F_{v}^{2} u_{z} \frac{\partial u_{z}}{\partial z}\right) & =-\frac{\partial P}{\partial z}-\rho^{\prime}, \\
\nabla_{h} \cdot \boldsymbol{u}_{h}+F_{v}^{2} \frac{\partial u_{z}}{\partial z} & =0, \\
\frac{\partial \rho^{\prime}}{\partial t}+\boldsymbol{u}_{h} \cdot \nabla_{h} \rho^{\prime}+F_{v}^{2} u_{z} \frac{\partial \rho^{\prime}}{\partial z}-u_{z} & =0 .
\end{aligned}
$$

where $\boldsymbol{u}_{h}$ and $\nabla_{h}$ are the horizontal components of $\boldsymbol{u}$ and $\nabla$. In practice, it will be more useful to express the horizontal velocity in term of the vertical vorticity $\zeta=\left(\nabla \times \boldsymbol{u}_{h}\right) \boldsymbol{e}_{z}$ by taking the curl of $(2.5 a)$

$$
\frac{\partial \zeta}{\partial t}+\boldsymbol{u}_{h} \cdot \nabla_{h} \zeta-F_{v}^{2}\left[\zeta \frac{\partial u_{z}}{\partial z}-u_{z} \frac{\partial \zeta}{\partial z}+\left(\nabla_{h} u_{z} \times \frac{\partial \boldsymbol{u}_{h}}{\partial z}\right) \boldsymbol{e}_{z}\right]=0 .
$$

\subsection{Basic state: the Lamb-Chaplygin dipole}

Since the vortex pair measured experimentally closely resembles the Lamb-Chaplygin vortex pair (Billant \& Chomaz 2000a), we choose this exact solution of the Euler equations as the basic state for the stability analysis $\dagger$. In a co-moving frame, the

$\dagger$ Note that the Lamb-Chaplygin dipole is not an exact solution of the Navier-Stokes equations, even with the time decay factor $\exp \left(-\mu_{1}^{2} t / R e\right)$, because of a discontinuity in the radial derivative of the vorticity at $r=1$. However, several studies (see, for instance, the recent study by Sipp, Jacquin $\&$ Cossu 2000) and the resemblance with experimental dipoles seem to indicate that this dipole with an appropriate time decay factor is almost a solution of the Navier-Stokes equations so that the discontinuity is likely to be of minor importance. 


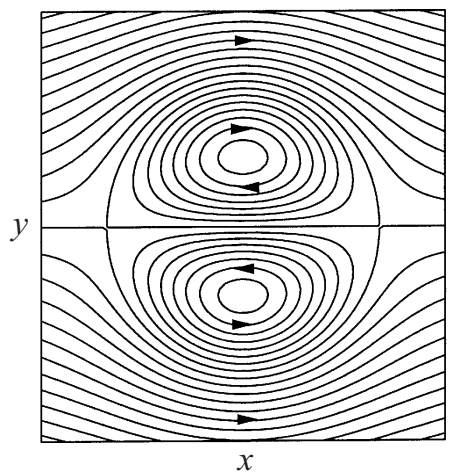

FIGURE 2. Streamlines of the Lamb-Chaplygin dipole.

non-dimensional streamfunction $\psi_{0}$ and vertical vorticity $\zeta_{0}=\Delta \psi_{0}$ of the LambChaplygin vortex pair are defined by (Lamb 1932; Batchelor 1967; Meleshko \& van Heijst 1994)

$$
\psi_{0}=-\frac{2}{\mu_{1} J_{0}\left(\mu_{1}\right)} J_{1}\left(\mu_{1} r\right) \sin \theta, \quad \zeta_{0}=-\mu_{1}^{2} \psi_{0},
$$

inside a circular region $r \leqslant 1$ and by

$$
\psi_{0}=-r\left(1-\frac{1}{r^{2}}\right) \sin \theta, \quad \zeta_{0}=0,
$$

outside $r>1 . J_{0}$ and $J_{1}$ are the Bessel functions of zero and first order and $\mu_{1}=3.8317$ is the first zero of $J_{1}$. The velocities and distance have been normalized by the propagating velocity $U$ and the radius of the dipole $R$. The streamlines of the Lamb-Chaplygin dipole are shown in figure 2. The corresponding two-dimensional velocity field is given by $\boldsymbol{u}_{h 0}=-\nabla \times\left(\psi_{0} \boldsymbol{e}_{z}\right)$.

\subsection{Linearized equations}

We subject this basic flow to infinitesimal perturbations denoted by a tilde

$$
\left(\boldsymbol{u}_{h} ; \zeta ; P ; \rho^{\prime} ; u_{z}\right)=\left(\boldsymbol{u}_{h 0} ; \Delta \psi_{0} ; P_{0} ; 0 ; 0\right)+\left(\tilde{\boldsymbol{u}}_{h} ; \tilde{\zeta} ; \tilde{P} ; \tilde{\rho}^{\prime} ; \tilde{u}_{z}\right),
$$

and linearize the equations of motion to obtain governing equations for the disturbance quantities

$$
\begin{aligned}
\frac{\partial \tilde{\zeta}}{\partial t}+\boldsymbol{u}_{h 0} \cdot \nabla_{h} \tilde{\zeta}+\tilde{\boldsymbol{u}}_{h} \cdot \nabla_{h} \Delta \psi_{0}-F_{v}^{2} \Delta \psi_{0} \frac{\partial \tilde{u}_{z}}{\partial z} & =0, \\
F_{h}^{2}\left(\frac{\partial \tilde{u}_{z}}{\partial t}+\boldsymbol{u}_{h 0} \cdot \nabla_{h} \tilde{u}_{z}\right) & =-\frac{\partial \tilde{P}}{\partial z}-\tilde{\rho}^{\prime}, \\
\nabla_{h} \cdot \tilde{\boldsymbol{u}}_{h}+F_{v}^{2} \frac{\partial \tilde{u}_{z}}{\partial z} & =0, \\
\frac{\partial \tilde{\rho}^{\prime}}{\partial t}+\boldsymbol{u}_{h 0} \cdot \nabla_{h} \tilde{\rho}^{\prime}-\tilde{u}_{z} & =0,
\end{aligned}
$$

with the following boundary conditions:

(i) As $r \rightarrow \infty$, the disturbances decay.

(ii) At $r=0$, the solution is non-singular.

(iii) The kinematic condition specifies that the boundary of the dipole continues 
to be the boundary. This is equivalent to requiring that the normal velocity $u_{r}$ be continuous at $r=1$.

(iv) The dynamic condition is that the pressure is continuous at the boundary of the dipole. Since the velocity of the dipole basic flow and its first derivative are continuous, this is tantamount to continuity of the tangential velocity of the perturbation $u_{\theta}$ at $r=1$.

Note that the stretching of the basic state's vertical vorticity $\zeta_{0}=\Delta \psi_{0}$ is the only three-dimensional effect which linearly affects the conservation of vertical vorticity in the linearized vertical vorticity equation $(2.9 a)$. In this respect, another way of writing (2.9a) is in terms of the linearized potential vorticity $\tilde{\Pi}=\tilde{\zeta}-F_{v}^{2} \Delta \Psi_{0} \partial \tilde{\rho}^{\prime} / \partial z$ which is conserved following the motion

$$
\frac{\partial \tilde{\Pi}}{\partial t}+\boldsymbol{u}_{h 0} \cdot \nabla_{h} \tilde{\Pi}+\tilde{\boldsymbol{u}}_{h} \cdot \nabla_{h} \Pi_{0}=0,
$$

where $\Pi_{0}=\Delta \Psi_{0}$ is the potential vorticity of the basic state. The potential vorticity will be useful in interpreting the instability mechanism.

\section{The asymptotic problem}

So far, the stability problem has been formulated generally. We now expand the perturbations in the small horizontal and vertical Froude numbers. It should be emphasized that the smallness of these two numbers has different meanings: $F_{h} \ll 1$ indicates that buoyancy dominates over inertial forces while $F_{v} \ll 1$ indicates that disturbances have a characteristic vertical wavelength $L_{v}$ long compared to the buoyancy length $U / N$. In other words, $F_{v}$ is the rescaled order of magnitude of the vertical wavenumber. Accordingly, the perturbation quantities should be expanded in both $F_{v}$ and $F_{h}$. For the sake of simplicity, we shall consider the vertical Froude number $F_{v}$ as the main small parameter of the expansion whereas the horizontal Froude number $F_{h}$ will be expressed in terms of $F_{v}$ and the aspect ratio $\delta=L_{v} / L_{h}$ through the relation $F_{h}=\delta F_{v}$. The aspect ratio $\delta$ will be formally considered as a free parameter of order one even if $F_{h}$ and $F_{v}$ have different magnitudes. This technical assumption does not detract from the rigour of the analysis provided that $F_{h}$ is small and leads to the same result as if a double series solution in terms of $F_{v}$ and $F_{h}$ were developed. The perturbations are thus expanded in the form

$$
\left(\tilde{\boldsymbol{u}}_{h} ; \tilde{\zeta} ; \tilde{P} ; \tilde{\rho}^{\prime} ; \tilde{u}_{z}\right)=\left(\tilde{\boldsymbol{u}}_{h 0} ; \tilde{\zeta}_{0} ; \tilde{P}_{0} ; \tilde{\rho}_{0}^{\prime} ; \tilde{u}_{z 0}\right)+F_{v}\left(\tilde{\boldsymbol{u}}_{h 1} ; \tilde{\zeta}_{1} ; \tilde{P}_{1} ; \tilde{\rho}_{1}^{\prime} ; \tilde{u}_{z 1}\right)+\cdots .
$$

Inserting this expansion into $(2.9 a-d)$, we obtain at leading order

$$
\begin{aligned}
\frac{\partial \tilde{\zeta}_{0}}{\partial t}+\boldsymbol{u}_{h 0} \cdot \nabla_{h} \tilde{\zeta}_{0}+\tilde{\boldsymbol{u}}_{h 0} \cdot \nabla_{h} \Delta \psi_{0} & =0, \\
\nabla_{h} \cdot \tilde{\boldsymbol{u}}_{h 0} & =0, \\
-\frac{\partial \tilde{P}_{0}}{\partial z}-\tilde{\rho}_{0}^{\prime} & =0, \\
\frac{\partial \tilde{\rho}_{0}^{\prime}}{\partial t}+\boldsymbol{u}_{h 0} \cdot \nabla_{h} \tilde{\rho}_{0}^{\prime}-\tilde{u}_{z 0} & =0 .
\end{aligned}
$$

As discussed in $\S 1$, the horizontal perturbation vorticity at the zeroth order satisfies linearized two-dimensional Euler equations with undetermined vertical dependence. Thus, the phase mode with the streamfunction $\tilde{\psi}_{0}=-\eta(z) \partial \psi_{0} / \partial y$ deriving from the translational invariance in the $y$-direction is a neutral solution of $(3.2 a-b)$. 
Because of the rotational invariance, a more general neutral perturbation solution of $(3.2 a-b)$ consists of both a translation $\eta$ in the $y$-direction and a rotation by an angle $\phi$ of the basic dipole

$$
\tilde{\psi}_{0}=-\eta(z, t) \frac{\partial \psi_{0}}{\partial y}+\phi(z, t) \frac{\partial}{\partial \theta}\left(\psi_{0}+y\right) .
$$

The term $y$ added to $\psi_{0}$ in the last derivative reflects the fact that only the basic flow in the laboratory frame is rotated and not the apparent flow resulting from the displacement of the reference of the frame. The last term in (3.3) corresponds merely to a dipole propagating at the velocity $\phi$ in the $y$-direction such that

$$
\begin{aligned}
& \frac{\partial \eta}{\partial t}=\phi, \\
& \frac{\partial \phi}{\partial t}=0 .
\end{aligned}
$$

The origin of the neutral perturbation (3.3) and the associated phase equations (3.4)(3.5) may be understood as follows: if the propagation direction of the dipole is turned slightly by an angle $\phi$ from the negative $x$-direction (figure 1), then the dipole begins to propagate in the $y$-direction at the velocity $\sin \phi \approx \phi$ to first order in $\phi$ since the propagating velocity is normalized to one (equation (3.4)). In contrast, the angle $\phi$ must remain constant to satisfy momentum conservation (equation (3.5)). This coupling between translational and rotational invariances is similar to the coupling between translational and Galilean invariances in the oscillatory instability of convection rolls (Coullet \& Fauve 1985; Fauve et al. 1987). A more formal way to derive the neutral perturbation (3.3) is by calculating, by means of a Galilean transformation, the flow of a dipole with arbitrary location $(x=\xi, y=\eta)$ and orientation $\phi$ expressed in the frame of reference moving with the unperturbed dipole with $\xi=\eta=\phi=0$. The infinitesimal neutral perturbation (3.3) is then obtained by considering the limiting case $\xi=0$ and $\eta, \phi \ll 1$.

However, the multiple-scale analysis requires that the phase variables $\eta$ and $\phi$ evolve on slow timescales $T_{1}=F_{v} t, T_{3}=F_{v}^{3} t, \ldots$ decoupled from the fast advective timescale $T_{0}=t$ and treated as independent variables

$$
\frac{\partial}{\partial t}=F_{v} \frac{\partial}{\partial T_{1}}+F_{v}^{3} \frac{\partial}{\partial T_{3}}+\cdots .
$$

Because of the relation (3.4) between $\eta$ and $\phi$, this slow evolution assumption effectively requires that the angle of rotation $\phi$ be one order smaller in $F_{v}$ than $\eta$. Therefore, at leading order, the perturbation solution of $(3.2 a-b)$ is simply taken as the translational mode

$$
\begin{gathered}
\tilde{\boldsymbol{u}}_{h 0}=-\eta\left(z, T_{1}, T_{3}\right) \frac{\partial \boldsymbol{u}_{h 0}}{\partial y} \\
\tilde{\zeta}_{0}=-\eta\left(z, T_{1}, T_{3}\right) \frac{\partial \Delta \psi_{0}}{\partial y}, \\
\tilde{P}_{0}=-\eta\left(z, T_{1}, T_{3}\right) \frac{\partial P_{0}}{\partial y}
\end{gathered}
$$

while the rotational mode will be obtained at next order $O\left(F_{v}\right)$ and could have been found without anticipating it from the structure of the invariance group. 

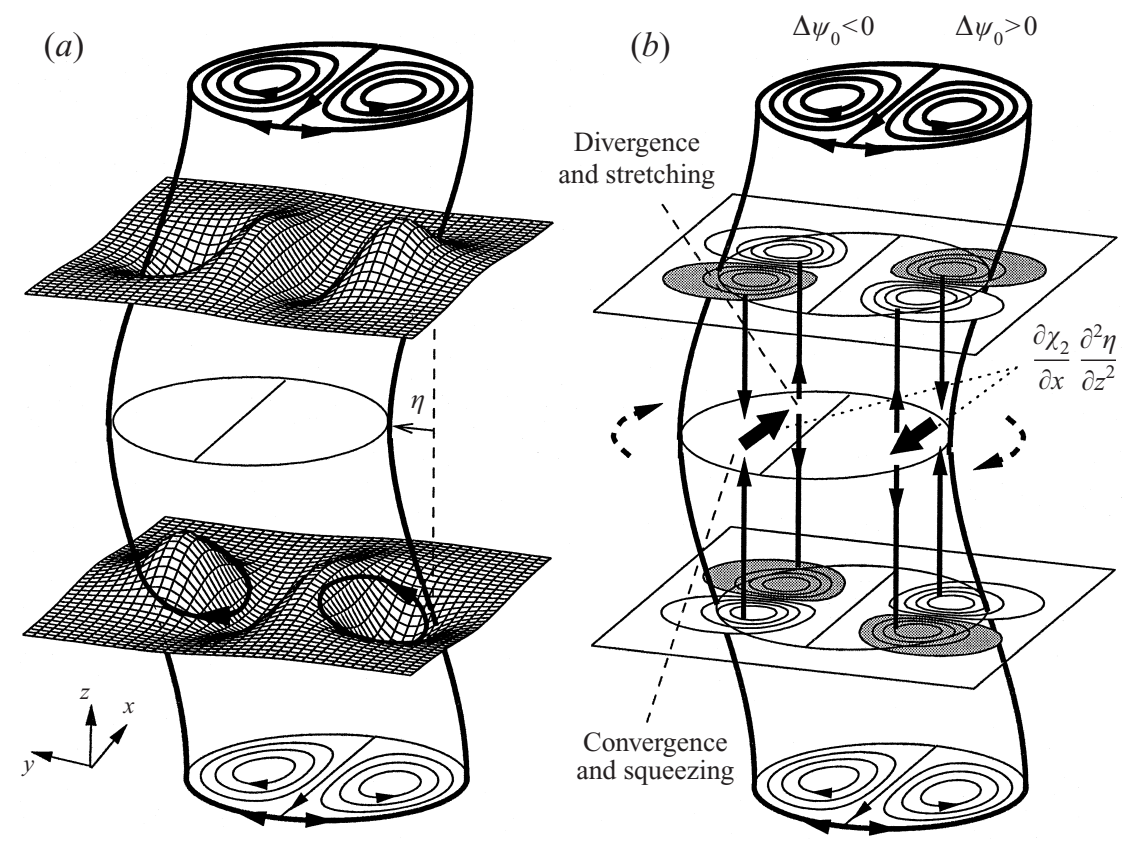

FiguRE 3. (a) Isosurfaces of the density field $\rho(z)+\tilde{\rho}_{0}^{\prime}$ where $\tilde{\rho}_{0}^{\prime}$ is given by (3.8), on either side of a sinusoidal bend $\eta$ in the $y$-direction. The amplitude of the deformations is arbitrary and has been exaggerated. The vortex pair propagates toward the reader. (b) Contours of the vertical velocity $\tilde{u}_{z 0}$ given by (3.9) owing to the isopycnal deformation shown in (a) Shaded regions indicate downward motions. The vertical arrows show the direction of the vertical velocity. The larger horizontal arrows indicate the direction of the potential flow along the $x$-axis that is generated at order $F_{v}^{2}$ in order to satisfy mass conservation. As established in $\S 3.2$, both this potential flow and the stretching of the dipole vertical vorticity by the vertical velocity impart a rotation to the middle dipole so that it turns clockwise. Owing to the propagation of the dipole, this twist tends to increase the initial $y$-displacement so that it will increase further in an unstable fashion.

We focus on these particular perturbations because they resemble the basic motions characterizing the zigzag instability in the experiment. Of course, there exist other phase modes deriving from the translational invariance in the $x$-direction or from the existence of parameters, such as the dipole radius and travelling speed along the $x$-axis, describing continuously the family of solutions $\dagger$. However, these modes are entirely decoupled from those considered in (3.3). This decoupling arises simply because normal modes separate into two independent classes: symmetric and antisymmetric in the $y$-direction, since the basic flow is symmetric in the $y$-direction. It turns out that the perturbation (3.3) is antisymmetric while the other phase modes are symmetric.

With the solution $(3.7 a-c)$, the density perturbation $\rho_{0}^{\prime}$ is next obtained from the hydrostatic balance $(3.2 c)$

$$
\tilde{\rho}_{0}^{\prime}=\frac{\partial P_{0}}{\partial y} \frac{\partial \eta}{\partial z}
$$

$\dagger$ In contrast with infinite periodic patterns, the neutral modes which consist of a uniform velocity field and derive from the Galilean invariance are not considered here because they do not satisfy the requirement that the perturbation velocity decays at infinity. The fact that the basic dipole is spatially localized is responsible for this difference. 
which inserted in the density equation (3.2d) yields the vertical velocity

$$
\tilde{u}_{z 0}=\nabla_{h} \cdot\left(\frac{\partial P_{0}}{\partial y} \boldsymbol{u}_{h 0}\right) \frac{\partial \eta}{\partial z},
$$

Figure 3(a) illustrates this leading-order dynamic and will allow us to easily understand the next order effects. Two isopycnal surfaces $\bar{\rho}(z)+\tilde{\rho}_{0}^{\prime}=$ constant are depicted on either side of a sinusoidal bend $\eta=\epsilon \sin (2 \pi z / \lambda)$ of the vortex pair. These surfaces are distorted by hydrostatic balance toward negative anomalies of pressure produced by the bend of the low-pressure vortex cores. For instance, the left-hand part of the lower isopycnal surface is brought upward because, above, the left-hand vortex is displaced to the left and, below, to the right.

To conserve density, fluid parcels are constrained to follow these distorted isopycnal surfaces. Therefore, a vertical velocity $(3.2 d)$ is created reflecting the fact that the fluid parcels, rotating around the vortex centres, rise and fall following the 'peaks' and 'valleys' of the isopycnal surfaces. Some examples of trajectories are sketched on the lower isopycnal surface in figure 3(a). Vertical velocity contours at the same level as the isopycnal surfaces are shown in figure $3(b)$. These contours display a quadrupole structure. By the above reasoning, the direction of the vertical velocity, sketched by vertical arrows, can be directly deduced from figure $3(a)$. The direction of the vertical velocity is reversed for the upper and lower contours since isopycnal deformations are opposite. At order $O\left(F_{v}^{2}\right)(\S 3.2)$, this symmetric vertical velocity field will stretch the vertical vorticity of the basic state and induce secondary horizontal motions in order to satisfy mass conservation. These effects turn out to be at the origin of the instability.

\subsection{Order $F_{v}$ problem}

Equating terms of order $F_{v}$ gives

$$
\begin{aligned}
\boldsymbol{u}_{h 0} \cdot \nabla_{h} \tilde{\zeta}_{1}+\tilde{\boldsymbol{u}}_{h 1} \cdot \nabla_{h} \Delta \psi_{0} & =\frac{\partial \Delta \psi_{0}}{\partial y} \frac{\partial \eta}{\partial T_{1}}, \\
\nabla_{h} \cdot \tilde{\boldsymbol{u}}_{h 1} & =0, \\
0 & =-\frac{\partial \tilde{P}_{1}}{\partial z}-\tilde{\rho}_{1}^{\prime}, \\
\boldsymbol{u}_{h 0} \cdot \nabla_{h} \tilde{\rho}_{1}^{\prime}-\tilde{u}_{z 1} & =-\frac{\partial \tilde{\rho}_{0}^{\prime}}{\partial T_{1}} .
\end{aligned}
$$

The solution to $(3.10 a-b)$ is the phase mode associated with the rotational invariance

$$
\begin{aligned}
\tilde{\boldsymbol{u}}_{h 1} & =-\frac{\partial \eta}{\partial T_{1}} \nabla \times\left[\left(\frac{\partial}{\partial \theta}\left(\psi_{0}+y\right)\right) \boldsymbol{e}_{z}\right], \\
\tilde{\zeta}_{1} & =\frac{\partial \eta}{\partial T_{1}} \frac{\partial \Delta \psi_{0}}{\partial \theta} \\
\tilde{P}_{1} & =\frac{\partial \eta}{\partial T_{1}} \frac{\partial P_{0}}{\partial \theta} .
\end{aligned}
$$

Thus, the perturbation at order $O\left(F_{v}\right)$ corresponds simply to a rotation of the basic flow by an angle $\phi_{1}$ defined by

$$
\frac{\partial \eta}{\partial T_{1}}=\phi_{1}
$$



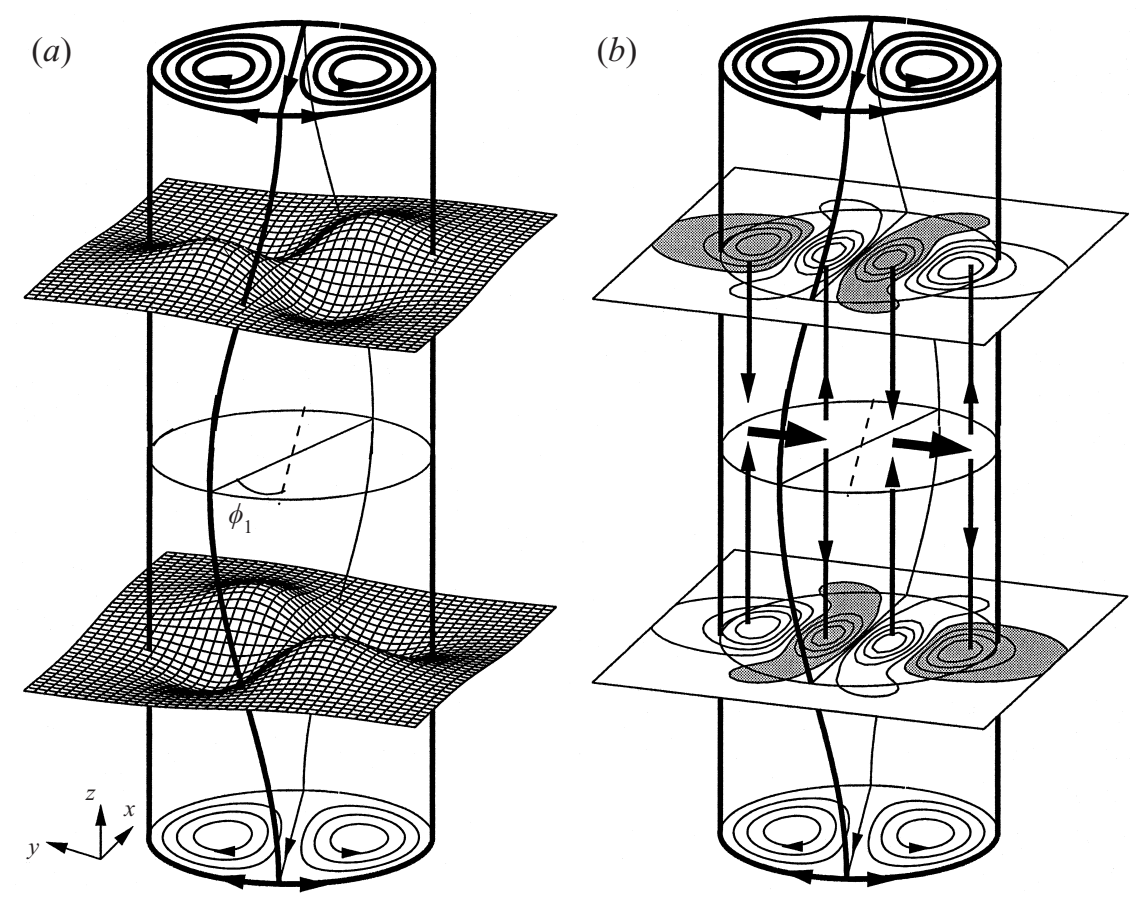

FigURE 4. (a) Isosurfaces of the density field $\rho(z)+\tilde{\rho}_{1}^{\prime}$ where $\tilde{\rho}_{1}^{\prime}$ is given by (3.13) above and below a sinusoidal twist $\phi_{1}$. The amplitudes of the deformations is arbitrary and have been exaggerated. The vortex pair is propagating toward the reader. $(b)$ Contours of the vertical velocity $\tilde{u}_{z 1}$ given by (3.14). Shaded regions indicate downward motions. The vertical arrows show the direction of the vertical velocity. The larger horizontal arrows indicate the direction of the potential flow along the $y$-axis that is generated at order $F_{v}^{3}$ in order to satisfy the divergence equation. Both this potential flow and the stretching of the dipole vertical vorticity by the vertical velocity tend to displace the middle dipole in the negative $y$-direction. Thus, the induced motions reduce the effect of the twist perturbation which, in contrast, generates a slight component of propagation in the positive $y$-direction. The induced motions in the case of a twist perturbation are therefore stabilizing, in contrast with the case of a bend perturbation.

The definition of the phase variable $\phi_{1}$ is only a convenient notation which is not at all imposed by solvability conditions. Its advantage is that it distinguishes the different effects due to the bend and the twist of the vortex pair and will provide a clearer physical understanding of the instability mechanism.

As was the case for the previous order, the hydrostatic equation $(3.10 c)$ gives next the density perturbation, using the definition (3.12),

$$
\tilde{\rho}_{1}^{\prime}=-\frac{\partial P_{0}}{\partial \theta} \frac{\partial \phi_{1}}{\partial z}
$$

As in figure 3(a), two isopycnal surfaces, perturbed only by $\rho_{1}^{\prime}$ are displayed in figure $4(a)$ on either side of a small sinusoidal twist of the vortex pair $\phi_{1}=\epsilon \sin (2 \pi z / \lambda)$. Their deformations are again due to the horizontal displacement of the low pressure vortex cores. The vertical velocity (figure $4 b$ ) is obtained from the density equation (3.10d), using (3.8) and (3.13),

$$
u_{z 1}=\left[-\nabla_{h} \cdot\left(\frac{\partial P_{0}}{\partial \theta} \boldsymbol{u}_{h 0}\right)+\frac{\partial P_{0}}{\partial y}\right] \frac{\partial \phi_{1}}{\partial z} .
$$


The first term in (3.14) is the vertical velocity necessary for fluid parcels moving with the basic horizontal velocity to follow the undulations of the isopycnal surfaces owing to the twisting of the vortex pair (figure $4 a$ ). As indicated by the vertical arrows, a squeezing on the left and a stretching on the right are produced at the mid-level of the left vortex. The vertical velocity in the right vortex is spatially distributed as in the left vortex. The second contribution in (3.14) comes from the slow evolution of the zeroth-order density perturbation $\tilde{\rho}_{0}^{\prime}$ over the timescale $T_{1}$ in $(3.10 d)$. If the bending deformation is amplifying, i.e. if $\partial \eta / \partial T_{1}=\phi_{1}>0$, the isopycnal distortions shown on figure 3(a) will steepen. Therefore, this term produces also a squeezing on the left and a stretching on the right at the midlevel of each vortex. As for the previous order, we anticipate that, at order $F_{v}^{3}$, this vertical velocity field will stretch the basic state vertical velocity in order to conserve potential vorticity and produce a horizontal flow so as to satisfy mass conservation.

\subsection{Order $F_{v}^{2}$ problem}

At the order $F_{v}^{2}$ of approximation, we have

$$
\begin{aligned}
\boldsymbol{u}_{h 0} \cdot \nabla_{h} \tilde{\zeta}_{2}+\tilde{\boldsymbol{u}}_{h 2} \cdot \nabla_{h} \Delta \psi_{0} & =\Delta \psi_{0} \frac{\partial \tilde{u}_{z 0}}{\partial z}-\frac{\partial \tilde{\zeta}_{1}}{\partial T_{1}}, \\
\nabla_{h} \cdot \tilde{\boldsymbol{u}}_{h 2}+\frac{\partial \tilde{u}_{z 0}}{\partial z} & =0 \\
\delta^{2}\left(\boldsymbol{u}_{h 0} \cdot \nabla_{h} \tilde{u}_{z 0}\right) & =-\frac{\partial \tilde{P}_{2}}{\partial z}-\tilde{\rho}_{2}^{\prime}, \\
\boldsymbol{u}_{h 0} \cdot \nabla_{h} \tilde{\rho}_{2}^{\prime}-\tilde{u}_{z 2} & =-\frac{\partial \tilde{\rho}_{1}^{\prime}}{\partial T_{1}} .
\end{aligned}
$$

Contrary to previous orders, non-trivial forcing terms appear in the vertical vorticity and divergence equations $(3.15 a-b)$. The zeroth-order vertical velocity stretches the basic-state vorticity (first term on the right-hand side of $(3.15 a)$ ) and appears as a forcing term in the divergence equation $(3.15 b)$.

In order to solve $(3.15 a-b)$, the horizontal velocity can be first separated into rotational and irrotational components with a streamfunction $\psi_{2}\left(r, \theta, z, T_{1}, T_{3}\right)$ and a potential function $\chi_{2}(r, \theta)$ in the form

$$
\tilde{\boldsymbol{u}}_{h 2}=-\nabla \times\left(\psi_{2} \boldsymbol{e}_{z}\right)+\nabla_{h} \chi_{2} \frac{\partial^{2} \eta}{\partial z^{2}} .
$$

In this case, using expression (3.9) for the vertical velocity $\tilde{u}_{z 0}$, the divergence equation (3.15b) becomes:

$$
\Delta_{h} \chi_{2}=-\nabla_{h} \cdot\left(\frac{\partial P_{0}}{\partial y} \boldsymbol{u}_{h 0}\right)=D_{2} .
$$

The function $D_{2}$ is written out in Appendix D. The solution is of the form

$$
\chi_{2}(r, \theta)=\chi_{2}^{(2)}(r) \sin 2 \theta+\chi_{2}^{(4)}(r) \sin 4 \theta,
$$

where the potential functions $\chi_{2}^{(2)}$ and $\chi_{2}^{(4)}$ are computed numerically with the boundary conditions (i)-(iv) (see $\S 2.4$ ), i.e. the velocity $\nabla_{h} \chi_{2}$ vanishes as $r \rightarrow \infty$, is non-singular at $r=0$ and continuous at $r=1$. The functions $\chi_{2}^{(2)}$ and $\chi_{2}^{(4)}$ are plotted in figure 5 . These functions are mainly confined to the rotational region $r \leqslant 1$ and decay in the potential region.

The streamfunction $\psi_{2}$ is next determined by substituting the expression (3.16) in 


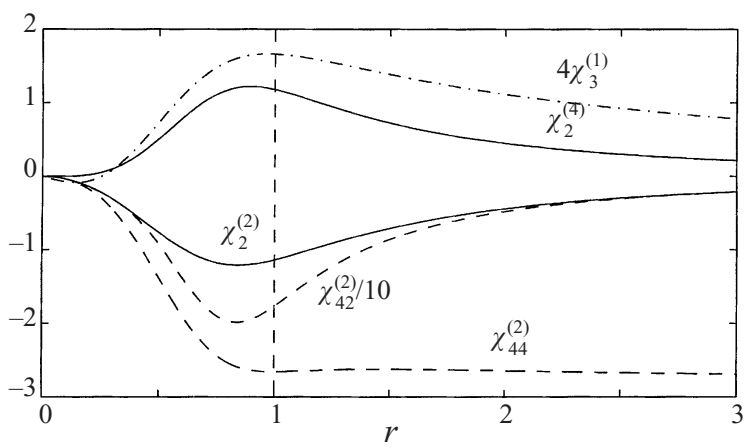

FIGURE 5. The potential functions $\chi_{2}^{(2)}(r)$ and $\chi_{2}^{(4)}(r)$ determined by (3.18), $\chi_{3}^{(1)}(r)$ determined by (C 3$)$, $\chi_{42}^{(2)}(r)$ determined by $(\mathrm{C} 20)$ and $\chi_{44}^{(2)}(r)$ determined by (C 19). The dashed vertical line indicates the boundary between the rotational and potential regions.

the equation for the vertical vorticity (3.15a), using (3.9), (3.11b) and (3.12)

$$
\begin{array}{ll}
L\left(\psi_{2}\right)=-\nabla_{h} \cdot\left(\Delta \psi_{0} \nabla_{h} \chi_{2}\right) \frac{\partial^{2} \eta}{\partial z^{2}}-\frac{\partial \Delta \psi_{0}}{\partial \theta} \frac{\partial \phi_{1}}{\partial T_{1}} & (r \leqslant 1), \\
\Delta_{h} \psi_{2}=0 & (r>1),
\end{array}
$$

with the boundary conditions (i)-(iv) and where $L$ is given by

$$
L\left(\psi_{2}\right) \equiv J\left(\psi_{0}, \Delta_{h} \psi_{2}+\mu_{1}^{2} \psi_{2}\right) \equiv \frac{1}{r} \frac{\partial \psi_{0}}{\partial r} \frac{\partial\left(\Delta_{h} \psi_{2}+\mu_{1}^{2} \psi_{2}\right)}{\partial \theta}-\frac{1}{r} \frac{\partial \psi_{0}}{\partial \theta} \frac{\partial\left(\Delta_{h} \psi_{2}+\mu_{1}^{2} \psi_{2}\right)}{\partial r},
$$

where $J$ denotes the Jacobian. The operator $L$ corresponds to the advection of the basic state's vertical vorticity (with $\Delta \psi_{0}=-\mu_{1}^{2} \psi_{0}$ ) by the horizontal rotational velocity of the perturbation and the advection of the perturbation vorticity by the basic flow. The right-hand side of (3.19) consists of forcing terms determined at previous order. The first forcing term is the sum of the stretching of the basic state vertical vorticity by the zeroth-order vertical velocity and the advection of the basic-state vorticity by the potential flow $\nabla_{h} \chi_{2}$.

Equation (3.19) will have a solution if and only if the right-hand side is orthogonal to the kernel of the adjoint operator $L^{\dagger}$ for the scalar product consisting of integration over the domain $r \leqslant 1$

$$
\langle f \mid g\rangle=\int_{0}^{2 \pi} \int_{0}^{1} f g r \mathrm{~d} r \mathrm{~d} \theta .
$$

where $f$ and $g$ are functions. We begin by treating the more general case of a steady basic state $\psi_{0}(x, y)$ such that $\Delta \psi_{0}=\mathscr{F}\left(\psi_{0}\right)$, without any particular choice for the functional $\mathscr{F}$, for which the corresponding operator $L$ is

$$
L(\psi)=J\left(\psi_{0}, \Delta_{h} \psi\right)-\mathscr{F}^{\prime}\left(\psi_{0}\right) J\left(\psi_{0}, \psi\right) .
$$

In Appendix $\mathrm{A}$, the adjoint operator $L^{\dagger}$ is obtained as

$$
L^{\dagger}(\tilde{\psi})=-\Delta_{h}\left(J\left(\psi_{0}, \tilde{\psi}\right)\right)+\mathscr{F}^{\prime}\left(\psi_{0}\right) J\left(\psi_{0}, \tilde{\psi}\right) .
$$

It is straightforward to see that any arbitrary function of $\psi_{0}$ is in the kernel of $L^{\dagger}$. It is almost as easy to show that the functions $\tilde{\psi}_{a}=r^{2}, \tilde{\psi}_{b}=x=r \cos \theta$ and 
$\tilde{\psi}_{c}=y=r \sin \theta$ are also solutions of $L^{\dagger}(\tilde{\psi})=0$ for any functional $\mathscr{F}$. For example, taking the case of $\tilde{\psi}_{b}$, we have $J\left(\psi_{0}, \tilde{\psi}_{b}\right)=-\partial \psi_{0} / \partial y$ so that

$$
L^{\dagger}\left(\tilde{\psi}_{b}\right)=\Delta_{h} \frac{\partial \psi_{0}}{\partial y}-\mathscr{F}^{\prime}\left(\psi_{0}\right) \frac{\partial \psi_{0}}{\partial y}=\frac{\partial}{\partial y}\left(\Delta_{h} \psi_{0}-\mathscr{F}\left(\psi_{0}\right)\right)=0 .
$$

These solutions reflect the translational and rotational invariances of the Euler equation. It can be demonstrated that for the Lamb-Chaplygin dipole, i.e. when $\mathscr{F}\left(\psi_{0}\right)=-\mu_{1}^{2} \psi_{0}$, there is no other null vector of the adjoint operator. Moreover, $\tilde{\psi}_{b}$, $\tilde{\psi}_{c}$ and any arbitrary function of $\psi_{0}$ satisfy the adjoint boundary conditions obtained in Appendix A, but $\tilde{\psi}_{a}$ does not. Therefore, denoting by $H$ the right-hand side of (3.19), the solvability conditions demand that

$$
\begin{gathered}
\left\langle\tilde{\psi}_{b} \mid H\right\rangle=0, \\
\left\langle\tilde{\psi}_{c} \mid H\right\rangle=0, \\
\left\langle\mathscr{G}\left(\psi_{0}\right) \mid H\right\rangle=0,
\end{gathered}
$$

where $\mathscr{G}$ stands for any arbitrary function of $\psi_{0}$. The two last conditions are automatically fulfilled in the case of (3.19) while the first condition will be satisfied only if

where the coefficient $D$ is

$$
\frac{\partial \phi_{1}}{\partial T_{1}}=D \frac{\partial^{2} \eta}{\partial z^{2}}
$$

$$
D=-\frac{\left\langle\tilde{\psi}_{b} \mid \nabla_{h} \cdot\left(\Delta \psi_{0} \nabla_{h} \chi_{2}\right)\right\rangle}{\left\langle\tilde{\psi}_{b} \mid \frac{\partial \Delta \psi_{0}}{\partial \theta}\right\rangle}
$$

After integration by parts and by evaluating the denominator, we find that

$$
D=-\frac{1}{2 \pi}\left\langle\Delta \psi_{0} \mid \frac{\partial \chi_{2}}{\partial x}\right\rangle \text {. }
$$

Another expression in terms of Bessel functions is obtained after further integration by parts and manipulations

$$
D=-\frac{3}{4}-\frac{1}{J_{0}^{4}\left(\mu_{1}\right)} \int_{0}^{\mu_{1}}\left(J_{2}^{\prime 2}(u) J_{1}^{2}(u)+4 J_{2}^{2}(u) J_{1}^{\prime 2}(u)\right) \frac{\mathrm{d} u}{u},
$$

from which we infer that the coefficient $D$ is negative. Numerical integration gives $D=-3.67$.

We pause for a moment to examine the equations that we have obtained so far over the slow time $T_{1}$. Using the definition (3.12) of $\phi_{1}$, (3.29) becomes

$$
\frac{\partial^{2} \eta}{\partial T_{1}^{2}}=D \frac{\partial^{2} \eta}{\partial z^{2}}
$$

Because $D$ is negative, perturbations of the form $\eta=\eta_{0} \mathrm{e}^{\sigma T_{1}+\mathrm{i} k z}$, where $k$ is the wavenumber along the $z$-axis, are amplified with a growth rate given by $\sigma^{2}=-D k^{2}$. This demonstrates that the columnar dipole is unstable for a dimensional wavelength that is long compared to $U / N$ in the limit $F_{h}=0$.

The physical mechanism of the instability can be easily understood from figure 3 . At zeroth order, we have seen that the small bending $\eta\left(z, T_{1}\right)$ of the vortex pair distorts the isopycnal surfaces by hydrostatic balance (figure $3 a$ ). In order that density be 
conserved, a slight vertical velocity is then generated (figure $3 b$ ). At order $F_{v}^{2}$, we see now that the convergence-divergence pattern implies a potential horizontal velocity $\nabla_{h} \chi_{2} \partial^{2} \eta / \partial z^{2}$ (indicated by large arrows in figure $3 b$ ) to satisfy mass conservation. As shown by dashed arrows in figure $3(b)$, this secondary flow tends to rotate the basic dipole at the midlevel in a clockwise direction by advection. In addition, the zeroth-order vertical velocity stretches and squeezes the basic-state vertical vorticity (figure $3 b$ ). In order to conserve potential vorticity, the squeezing-stretching pattern tends, respectively, to decrease and increase the magnitude of the vertical vorticity at the midlevel of the front and the rear of the left vortex. The situation is reversed for the right vortex. Hence, the tendency in the vertical vorticity equation $(3.15 a)$ resulting from this effect is also a clockwise rotation of the basic dipole at the midlevel. The two effects, namely the advection by the secondary potential velocity field and the stretching-squeezing of the basic vertical vorticity, therefore act in the same way and indeed appear together in the expression (3.31) of the coefficient $D$. This convenient expression indicates that $D$ is negative when $\partial \chi_{2} / \partial x$ is of the same sign as the vorticity distribution $\Delta \psi_{0}$ inside the dipole. Therefore, $D$ is negative when the net macroscopic effect of the velocity $\left(\partial \chi_{2} / \partial x\right)\left(\partial^{2} \eta / \partial z^{2}\right)$ is to advect the dipole so as to rotate it clockwise (counterclockwise) if $\partial^{2} \eta / \partial z^{2}$ is negative (positive); see figure $3 b$. Owing to the propagation of the dipole, this twist $\phi_{1}$ of the travelling direction tends to increase the initial displacement $\eta$ through (3.12) so that it will increase further in an unstable fashion.

At this level of approximation, the growth rate increases without bound with wavenumber. To see if stabilization might occur at high wavenumber, we shall proceed to the next orders. To this end, we return to the determination of $\psi_{2}$. Inserting the phase equation (3.33) in (3.19), we are now able to find $\psi_{2}$ since all solvability conditions are satisfied

$$
\left.\begin{array}{ll}
L\left(\psi_{2}\right)=-\left[\nabla_{h} \cdot\left(\Delta \psi_{0} \nabla_{h} \chi_{2}\right)+D \frac{\partial \Delta \psi_{0}}{\partial \theta}\right] \frac{\partial^{2} \eta}{\partial z^{2}} \equiv H_{2} \frac{\partial^{2} \eta}{\partial z^{2}} & (r \leqslant 1), \\
\Delta_{h} \psi_{2}=0 & (r>1) .
\end{array}\right\}
$$

In order to solve this equation, the streamfunction $\psi_{2}$ is written in the form

$$
\psi_{2}=\Psi_{2}(r, \theta) \frac{\partial^{2} \eta}{\partial z^{2}} .
$$

The first step is to find a function $G$ such that

$$
J\left(\psi_{0}, G\right)=H_{2},
$$

for $r \leqslant 1$, as in Appendix B.1. Then, an integral form of (3.34) is

$$
\left.\begin{array}{ll}
M\left(\Psi_{2}\right) \equiv \Delta_{h} \Psi_{2}+\mu_{1}^{2} \Psi_{2}=G+\mathscr{K}\left(\psi_{0}\right) & (r \leqslant 1), \\
\Delta_{h} \Psi_{2}=0 & (r>1),
\end{array}\right\}
$$

where $M$ is the Helmholtz operator and $\mathscr{K}\left(\psi_{0}\right)$ is any arbitrary function of $\psi_{0}$. The major difficulty arising in this equation is the choice of the free function $\mathscr{K}\left(\psi_{0}\right)$. In Appendix B.2, we demonstrate that $\mathscr{K}\left(\psi_{0}\right)$ is determined on the basis of a solvability condition at order $F_{v}^{3}$. Once $\mathscr{K}\left(\psi_{0}\right)$ has been determined, the Helmholtz equation (3.37) can be solved numerically by expanding the solution as a Fourier series

$$
\Psi_{2}(r, \theta)=\Psi_{2}^{(0)}(r)+\Psi_{2}^{(2)}(r) \cos 2 \theta+\Psi_{2}^{(4)}(r) \cos 4 \theta+\Psi_{2}^{(6)}(r) \cos 6 \theta \cdots .
$$

The first three Fourier functions $\Psi_{2}^{(0)}, \Psi_{2}^{(2)}$ and $\Psi_{2}^{(4)}$ are shown in figure 6. The 


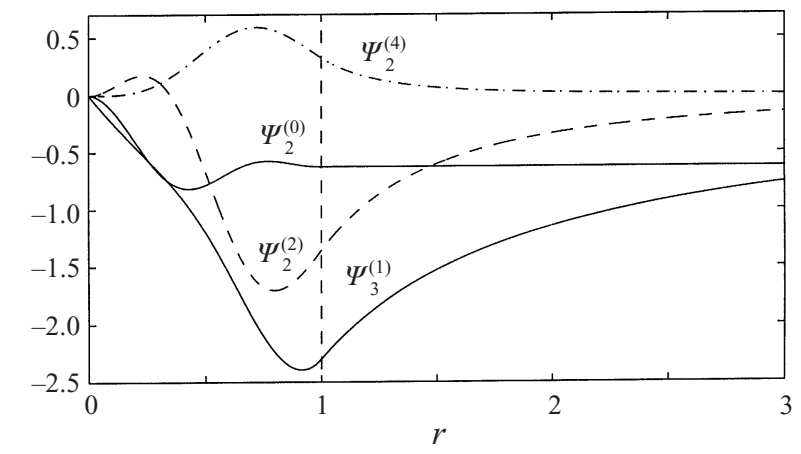

FIGURE 6. Streamfunctions at order $F_{v}^{2}: \Psi_{2}^{(0)}, \Psi_{2}^{(2)}, \Psi_{2}^{(4)}$ defined in (3.38) and at order $F_{v}^{3}: \Psi_{3}^{(1)}$ defined in (C9). The dashed vertical line indicates the boundary between the rotational and potential regions.

following functions $\Psi_{2}^{(2 n)},(n \geqslant 3)$ have also been determined but it turns out that they are very small (i.e. $\max \left(\Psi_{2}^{(6)}\right)=0.025, \max \left(\Psi_{2}^{(8)}\right)=0.009$ ) so that they are not plotted. This is because the forcing term in (3.37) contains mainly the components $(n<3)$. The following forcing components $(n \geqslant 3)$ are very small and come only from the function $\mathscr{K}\left(\psi_{0}\right)$.

The pressure $\tilde{P}_{2}$ is written in the form

$$
\tilde{P}_{2}=\Pi_{2}(r, \theta) \frac{\partial^{2} \eta}{\partial z^{2}},
$$

and using (3.9), the density $\tilde{\rho}_{2}^{\prime}$ is obtained from the vertical momentum equation $(3.15 c)$

$$
\tilde{\rho}_{2}^{\prime}=-\Pi_{2} \frac{\partial^{3} \eta}{\partial z^{3}}-\delta^{2} \boldsymbol{u}_{h 0} \cdot \nabla_{h}\left[\nabla_{h} \cdot\left(\frac{\partial P_{0}}{\partial y} \boldsymbol{u}_{h 0}\right)\right] \frac{\partial \eta}{\partial z} .
$$

The first term in (3.40) represents the hydrostatic adjustment of isopycnal surfaces to the pressure field $\tilde{P}_{2}$. Calculations show that this term will be stabilizing at order $O\left(F_{v}^{4}\right)$. The second term in (3.40) corresponds to the horizontal advection of the vertical velocity $\tilde{u}_{z 0}$ by the basic flow. It is easy to deduce that this term will be destabilizing at order $O\left(F_{v}^{4}\right)$.

The vertical velocity is next determined from the density equation $(3.15 d)$, using (3.13), (3.33) and (3.40)

$$
\tilde{u}_{z 2}=-\left[\nabla_{h} \cdot\left(\Pi_{2} \boldsymbol{u}_{h 0}\right)+D \frac{\partial P_{0}}{\partial \theta}\right] \frac{\partial^{3} \eta}{\partial z^{3}}-\delta^{2} \boldsymbol{u}_{h 0} \cdot \nabla_{h}\left[\boldsymbol{u}_{h 0} \cdot \nabla_{h}\left[\nabla_{h} \cdot\left(\frac{\partial P_{0}}{\partial y} \boldsymbol{u}_{h 0}\right)\right]\right] \frac{\partial \eta}{\partial z} .
$$

The first and third terms in (3.41) come from the advection of the density perturbation $\tilde{\rho}_{2}^{\prime}$ by the basic flow. The second term is the slow evolution of $\tilde{\rho}_{1}^{\prime}$ over the timescale $T_{1}$. Its effect can be easily inferred: if the twist of the vortex pair increases, i.e. if $\partial \phi_{1} / \partial T_{1}>0$, the isopycnal deformations shown in figure $4(a)$ will steepen. Therefore, at the midlevel, there will be a stretching at the front of the left-hand vortex and a squeezing at the rear. The situation is reversed for the right-hand vortex. Hence, the generated vertical velocities are opposite to those of figure $3(a)$ and will contribute to stabilizing effects. 


\subsection{Higher-order problems}

The perturbation problems up to order $O\left(F_{v}^{4}\right)$ are solved in Appendix $\mathrm{C}$ using the same method as for the second order, i.e. by separating the perturbation velocity into potential and rotational components. To find a solution to the $O\left(F_{v}^{4}\right)$ problem, the solvability conditions demand that

$$
\frac{\partial \phi_{1}}{\partial T_{3}}+\frac{\partial \phi_{3}}{\partial T_{1}}=g_{1} \delta^{2} \frac{\partial^{2} \eta}{\partial z^{2}}+g_{2} \frac{\partial^{4} \eta}{\partial z^{4}},
$$

where the coefficients are $g_{1}=-56.4$ and $g_{2}=-16.1$ and where we have defined, as for the first order, a third phase variable $\phi_{3}$

$$
\frac{\partial \eta}{\partial T_{3}}=\phi_{3}
$$

The coefficient $g_{2}$ is negative, indicating that the fourth vertical derivative of $\eta$ provides stabilizing effects. Although there are several complex effects contributing to this coefficient, one simple stabilizing effect can be intuitively understood from figure 4. As for the second order, the convergence-divergence pattern resulting from the twist of the vortex pair at order $O\left(F_{v}\right)$ produces at order $O\left(F_{v}^{3}\right)$ a potential horizontal velocity field. As indicated by large horizontal arrows (figure $4 b$ ), this induced horizontal potential flow tends to advect the midlevel dipole toward the right. The local stretching and squeezing of the basic-state vorticity also generate a tendency in the vertical vorticity equation which results in a shift of the midlevel dipole toward the right. This tends to reduce the effect of the twist which, on the contrary, shifts the dipole leftward (figure $4 b$ ).

\section{Linear phase dynamics analysis}

The slow time variables are first rescaled and expressed in terms of a single time variable $t$ such that

$$
\frac{\partial}{\partial t}=F_{v} \frac{\partial}{\partial T_{1}}+F_{v}^{3} \frac{\partial}{\partial T_{3}} .
$$

Then, (3.12), (3.29), (3.42) and (3.43) can be combined to give

$$
\begin{gathered}
\frac{\partial \eta}{\partial t}=\phi, \\
\frac{\partial \phi}{\partial t}=F_{v}^{2}\left(D+F_{h}^{2} g_{1}\right) \frac{\partial^{2} \eta}{\partial z^{2}}+F_{v}^{4} g_{2} \frac{\partial^{4} \eta}{\partial z^{4}}+O\left(F_{v}^{6}, F_{v}^{4} F_{h}^{2}, F_{v}^{2} F_{h}^{4}\right),
\end{gathered}
$$

where $D=-3.67, \quad g_{1}=-56.4, \quad g_{2}=-16.1$. These phase equations are expected to be valid when $F_{h}$ and $F_{v}$ are sufficiently small. They resemble closely those describing the original zigzag instability of convective rolls (Pomeau \& Manneville 1979) except that, because of the time-reversibility of the Euler equations, the dynamic is second order in time instead of first order. This similarity is not fortuitous but rooted in the symmetry breaking of the instability. Following this consideration and the fact that the vortex pair indeed exhibits a zigzag deformation, we have also used the term zigzag to describe the present instability.

The structure of (4.2)-(4.3) in the two-dimensional limit $F_{v}=0$ derives from the coupling between translational and rotational invariances (see $\S 3$ ). The additional terms in the right-hand side of (4.3) are in powers of the small horizontal and vertical 
(a)

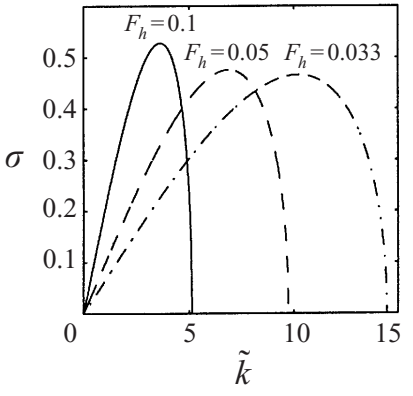

(b)

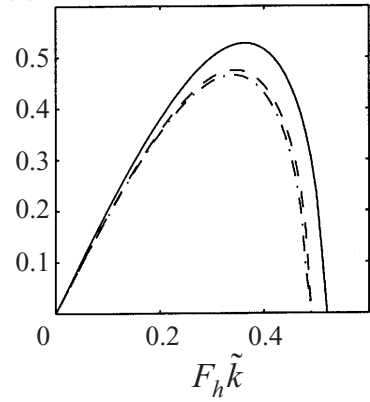

FIGURE 7. Non-dimensional growth rate $\sigma(a)$ as a function of the non-dimensional vertical wavenumber $\tilde{k}$ and $(b)$ as a function of $F_{h} \tilde{k}$ for,$- F_{h}=0.1 ;---, F_{h}=0.05 ;-\cdot-, F_{h}=0.033$.

Froude numbers and correspond to weak three-dimensional effects obtained by the multiple-scale perturbation analysis. The form of (4.2)-(4.3) could have been deduced solely by symmetry considerations. We first observe that the equations are invariant under the transformation $\eta \rightarrow \eta+c$ where $c$ is a constant. This reflects translational invariance. The invariance under the transformations $(\phi \rightarrow \phi+c, \eta \rightarrow \eta+c t)$, although reminiscent of the Galilean invariance, derives from the fact that the dipole travelling direction and speed can be chosen arbitrarily. The invariance under the transformations $(\eta \rightarrow-\eta, \phi \rightarrow-\phi)$ and $z \rightarrow-z$ reflect space-reflection symmetries. Finally, the invariance under $(t \rightarrow-t, \eta \rightarrow-\eta)$ reflects time reversibility of the Euler equations. Although these symmetry constraints could allow us to guess the form of the nonlinear terms, we focus herein on the linear dynamic described by (4.2)-(4.3).

\subsection{Linear stability analysis}

We investigate the linear stability of (4.2)-(4.3) with respect to perturbations of the form

$$
(\eta, \phi)=\left(\eta_{0}, \phi_{0}\right) \mathrm{e}^{\sigma t+\mathrm{i} k z},
$$

which leads to the dispersion relation

$$
\sigma^{2}=-\left(D+g_{1} F_{h}^{2}\right) F_{v}^{2} k^{2}+g_{2} F_{v}^{4} k^{4} .
$$

Sufficiently long waves $\left(F_{v} \ll 1\right)$ are always unstable because the coefficients $D$ and $g_{1}$ are negative. There is, however, a stabilization at large wavenumber since $g_{2}$ is negative.

To study quantitatively (4.5), it is convenient to abandon $L_{v}$, which measures the order of magnitude of the vertical wavelength. We now scale the vertical wavenumber by the horizontal lengthscale $L_{h}=R$. This new dimensionless wavenumber is denoted by $\tilde{k}$ where $\tilde{k}=k R / L_{v}, k$ being the previous wavenumber non-dimensionalized by $L_{v}$. In this case, (4.5) becomes

$$
\sigma^{2}=-\left(D+g_{1} F_{h}^{2}\right) F_{h}^{2} \tilde{k}^{2}+g_{2} F_{h}^{4} \tilde{k}^{4} .
$$

This dispersion relation is quantitatively valid only for $F_{h} \ll 1$ and $\tilde{k} \ll 1 / F_{h}$, i.e. when the wavelength is long compared to $U / N$. In order to study the qualitative behaviour of this approximation, the growth rate of the whole unstable wavenumber bandwidth has been plotted in figure 7(a) for various small horizontal Froude numbers. The most striking feature is that the growth rate curves are shifted to high wavenumbers as $F_{h}$ decreases. The maximal growth rate does not decrease with $F_{h}$ but remains almost 
constant. These properties are an immediate consequence of the fact that $\tilde{k}$ appears in (4.6) always associated to $F_{h}$ in the form $\left(F_{h} \tilde{k}\right)^{m}$ multiplied by $O(1)$ coefficients with $O\left(F_{h}^{2}\right)$ corrections. Thus, when represented as a function of $F_{h} \tilde{k}$, the growth rate curves almost gather on a single curve, as shown in figure $7(b)$. The similarity is not perfect because of the presence of the term $g_{1} F_{h}^{2}$ in (4.6). This term is, however, small in comparison with the coefficient $D$ since $F_{h} \ll 1$.

Therefore, (4.6) predicts that the wavenumber $\tilde{k}_{m}$ of the most amplified disturbance

$$
\tilde{k}_{m}=\frac{1}{F_{h}} \sqrt{\frac{D+g_{1} F_{h}^{2}}{2 g_{2}}},
$$

is proportional to $1 / F_{h}$ as $F_{h} \rightarrow 0$, while its growth rate

$$
\sigma_{m}=-\frac{D+g_{1} F_{h}^{2}}{2 \sqrt{-g_{2}}}
$$

is independent of $F_{h}$ when $F_{h} \rightarrow 0$. These two expressions are, of course, not quantitatively valid since they violate the long-wavelength assumption $\tilde{k} \ll 1 / F_{h}$ used to obtain (4.6). In the next section, we show, however, that the predictions $\tilde{k}_{m} \propto 1 / F_{h}$ and $\sigma_{m}=$ constant when $F_{h} \rightarrow 0$ are qualitatively correct. Only the proportionality constants are not valid.

\subsection{Extension of the dispersion relation}

A careful inspection of the nature of the expansion shows that if we had carried it out for an infinite number of terms, we would have obtained a power series of the form

$$
\begin{aligned}
\sigma^{2}= & \sum_{n=1}^{\infty} a_{2 n}\left(F_{h} \tilde{k}\right)^{2 n}+\sum_{n=3}^{\infty} a_{2 n}^{\prime}\left(F_{h} \tilde{k}\right)^{2 n} \ln \left(F_{h} \tilde{k}\right) \\
& +F_{h}^{2}\left(\sum_{n=1}^{\infty} b_{2 n}\left(F_{h} \tilde{k}\right)^{2 n}+\sum_{n=4}^{\infty} b_{2 n}^{\prime}\left(F_{h} \tilde{k}\right)^{2 n} \ln \left(F_{h} \tilde{k}\right)\right)+F_{h}^{4} \sum_{n=1}^{\infty} c_{2 n}\left(F_{h} \tilde{k}\right)^{2 n}+\cdots
\end{aligned}
$$

where we have determined the first coefficients of this serie: $a_{2}=-D, a_{4}=g_{2}, b_{2}=g_{1}$. (It turns out that terms of the form $\left(F_{h} \tilde{k}\right)^{2 n} \ln \left(F_{h} \tilde{k}\right)$ would arise for $n \geqslant 3$ in order to make the expansion uniformly asymptotic at large $r$.) The series can be more conveniently written in terms of functions $f_{i}$ of $F_{h} \tilde{k}$

$$
\sigma^{2}=f_{0}\left(F_{h} \tilde{k}\right)+F_{h}^{2} f_{2}\left(F_{h} \tilde{k}\right)+F_{h}^{4} f_{4}\left(F_{h} \tilde{k}\right)+\cdots,
$$

which indicates that, when $F_{h} \rightarrow 0$, the growth rate becomes a function of $F_{h} \tilde{k}$ only

$$
\sigma^{2}=f_{0}\left(F_{h} \tilde{k}\right)+O\left(F_{h}^{2}\right)
$$

as in the approximation (4.6). Therefore, the self-similarity of the growth rate with respect to the variable $F_{h} \tilde{k}$ is not a spurious consequence of the long-wavelength approximation. According to (4.11), the wavenumber of the most amplified disturbance will be in the limit $F_{h} \rightarrow 0$

$$
\tilde{k}_{m}=d_{m} / F_{h},
$$

where $d_{m}$ is the value at which the function $f_{0}$ is maximal. The corresponding maximal growth rate (non-dimensionalized by the turnover frequency $U / L_{h}$ ) becomes 
independent of the horizontal Froude number

$$
\sigma_{m}^{2}=f_{0}\left(d_{m}\right)
$$

The independence of the growth rate of the three-dimensional instability with respect to the Froude number is in agreement with the numerical simulations of forced stratified turbulence by Herring \& Métais (1989). As discussed in $\S 1$, a turbulent flow, initially vertically uniform, was forced two-dimensionally. Subsequently, a threedimensional perturbation was introduced. Despite the fact that the basic state differs widely from that considered here, this disturbance was also observed to grow exponentially at a rate independent of the Brunt-Väisälä frequency. Unfortunately, the vertical wavenumber was not reported in this study, so that it is unknown if it scales as $1 / F_{h}$. After the exponential growth regime, saturation occurred when a statistical equilibrium between forcing and dissipation was reached. At this stage, the flow was organized into decoupled horizontal layers as a result of the three-dimensional instability. However, in this regime, the layer thickness was no longer selected by the instability mechanism but was governed by the equilibrium between dissipation by vertical shearing and input of kinetic energy by the two-dimensional forcing. This final layer thickness was reported to be independent of the Brunt-Väisälä frequency but this result cannot be compared to the previous predictions for an unforced flow.

\subsection{Comparison with experiments}

The most compelling agreement with experiment concerns the qualitative behaviour of the zigzag instability (Billant \& Chomaz 2000a). As observed experimentally, the unstable perturbation at leading orders consists both of a vertically modulated rotation $\phi(z, t)$ and translation $\eta(z, t)$ of the vortex pair perpendicular to the propagation direction.

In contrast, we do not expect agreement between the observed wavelength and the most amplified wavelength predicted by our asymptotic analysis since, as already stated, the long-wavelength hypothesis $F_{h} \widetilde{k} \ll 1$ is no longer valid for such wavelengths. Furthermore, the scaling $\lambda / R \propto F_{h}$ has not been observed in the experiments because the Froude-number range is narrow and the wavelength fluctuations are large. Nevertheless, we can check that the asymptotic analysis predicts the correct order of magnitude for the most amplified dimensional wavelength. In the experiment, the wavelength is about $\lambda / R \approx 1.7$ but fluctuates between 1.1 and 2.5 in the Froude-number range $0.13<F_{h}<0.21$. In this range, the most amplified wavelength $\lambda_{m} / R=18.6 F_{h} / \sqrt{1+15.4 F_{h}^{2}}$ obtained by the asymptotic analysis varies between $\lambda_{m} / R=2.2$ and $\lambda_{m} / R=3$. These values differ but are nonetheless of the same order of magnitude as the wavelength measured experimentally.

Moreover, viscous effects have not been taken into account in the asymptotic analysis while in the experiments, the initial Reynolds number is relatively low, between $R e=250$ and $R e=400$. Although the algebra is more complicated if a weak viscosity (i.e. $1 / R e=O\left(F_{v}^{2}\right)$ ) is taken into account in the asymptotic analysis, the final result is simple: the dispersion relation (4.6) becomes

$$
\left(\sigma+\frac{1}{R e}\left(\tilde{k}^{2}+\mu_{1}^{2}\right)\right)^{2}=-\left(D+g_{1} F_{h}^{2}\right) F_{h}^{2} \tilde{k}^{2}+g_{2} F_{h}^{4} \tilde{k}^{4},
$$

where $R e$ is the Reynolds number. As usual, the viscous diffusion of the basic state has been omitted to obtain (4.14). It should be mentioned, however, that because of the discontinuity in the radial derivative of the vorticity at $r=1$ of the Lamb-Chaplygin 

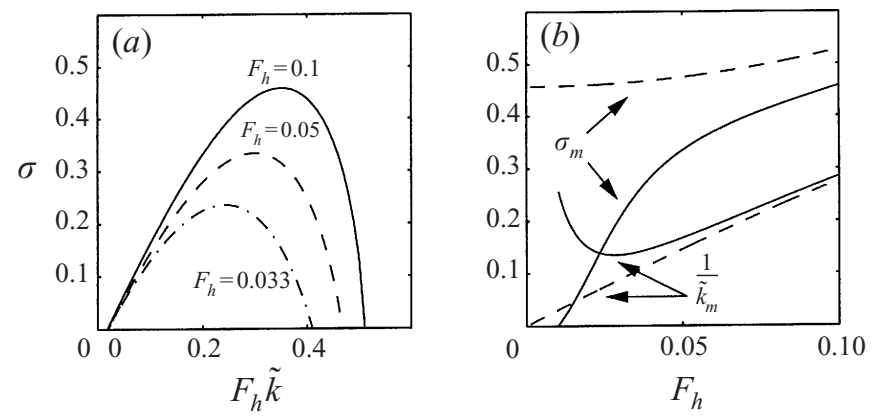

FIGURE 8. (a) Growth rate $\sigma$ given by (4.14) as a function of $F_{h} \tilde{k}$ for $R e=400$ and for the same Froude numbers as in figure 7. (b) Maximum growth rate $\sigma_{m}$ and inverse of the most amplified wavenumber $1 / \tilde{k}_{m}$ obtained from (4.14) as a function of $F_{h}$ for $R e=400(-)$ and for the inviscid case $(---)$.

dipole (see § 2.3 ), only the inviscid boundary conditions (iii)-(iv) (see § 2.4 ) have been applied to the perturbations at $r=1$. As testified by the physically reasonable form of the viscous correction obtained in (4.14), this simplification should not be detrimental at least to the qualitative features of the dispersion relation which are discussed below.

As seen from (4.14) and figure 8, viscous effects break the self-similarity for low Reynolds number and large wavenumber because the growth rate is no longer approximately a function of the single variable $F_{h} \tilde{k}$. In practice, inviscid stability curves will be unaffected by viscous effects only if the Reynolds number is large: $R e \gg 1 / F_{h}^{2}$. Figure $8(b)$ shows also that viscous effects introduce a Froude number threshold below which the instability is damped, as observed experimentally. For large Reynolds number, this critical Froude number varies as $F_{h c} \approx 2 \mu_{1} /(\sqrt{-D} R e)$. Slightly above $F_{h c}$, there is a significant decrease of the most amplified wavenumber relative to the inviscid case (figure $8 b$ ).

\section{Conclusions and discussion}

In this paper, we have performed a multiple-scale stability analysis of a columnar Lamb-Chaplygin vortex pair in a stratified fluid for small horizontal and vertical Froude numbers. The smallness of the horizontal Froude number means that buoyancy is dominant over inertial forces while $F_{v} \ll 1$ implies that disturbances have a long vertical wavelength compared to $U / N$. In the limit $F_{v} \rightarrow 0$, the zigzag instability has been shown to be a phase instability deriving from the breaking of translational invariance in the $y$-direction and rotational invariance in the horizontal plane. Periodically along the vertical, the columnar vortex pair will therefore be twisted and bent in the $y$-direction, i.e. perpendicularly to the travelling direction. These motions are those characterizing the zigzag instability observed in the experiments (Billant \& Chomaz 2000a).

The perturbation approach provides a clear physical understanding of the instability mechanism. If the columnar vortex pair is vertically bent in the $y$-direction, the resulting vertical pressure gradient distorts by hydrostatic balance the isopycnal surfaces. In response, a vertical velocity field is generated in order to conserve density. This vertical velocity field stretches the basic vertical vorticity and induces secondary horizontal motions in order that mass be conserved. These two effects induce a twisting of the vortex pair which modifies the travelling direction in such a way as to increase the initial bend, thus leading to instability. 
The long-wavelength dispersion relation indicates that the instability survives in the limit of strong stratification $F_{h} \rightarrow 0$. The maximal growth rate is predicted to scale as $U / L_{h}$, i.e. independent of the horizontal Froude number, and the most amplified dimensional vertical wavelength as $U / N$. Since the zigzag instability is observed experimentally to be responsible for the formation of decoupled horizontal layers with a thickness corresponding to the initial wavelength, the vertical Froude number in the resulting flow is such that $F_{v}=O(1)$ in contradiction to the assumption $F_{v} \ll 1$ of Riley et al. (1981) and Lilly (1983). Setting $F_{v}=1$ in the non-dimensional equations $(2.5 a-d)$ shows that the horizontal flow does not satisfy two-dimensional Euler equations at leading order in $F_{h}$ but instead the equations

$$
\begin{aligned}
\frac{\partial \boldsymbol{u}_{h}}{\partial t}+\boldsymbol{u}_{h} \cdot \nabla_{h} \boldsymbol{u}_{h}+u_{z} \frac{\partial \boldsymbol{u}_{h}}{\partial z} & =-\nabla_{h} P, \\
0 & =-\frac{\partial P}{\partial z}-\rho^{\prime}, \\
\nabla_{h} \cdot \boldsymbol{u}_{h}+\frac{\partial u_{z}}{\partial z} & =0, \\
\frac{\partial \rho^{\prime}}{\partial t}+\boldsymbol{u}_{h} \cdot \nabla_{h} \rho^{\prime}+u_{z} \frac{\partial \rho^{\prime}}{\partial z}-u_{z} & =0,
\end{aligned}
$$

which are similar to the primitive equations often used in weather forecasting and ocean modelling. The sole approximation allowed by $F_{h} \ll 1$ is the hydrostatic equilibrium in the vertical momentum equation $(5.1 b)$. The vertical velocity is still small, scaling as $F_{h} U$, but its smallness is exactly balanced by the largeness of vertical gradients $\partial / \partial z=O\left(1 / F_{h} L_{h}\right)$. The limit $F_{v} \rightarrow 0$ considered by Riley et al. (1981) and Lilly (1983) is singular because the thicknesses of the horizontal layers decrease as the stratification increases. Therefore, even if the motion is horizontal at leading order, vertical gradients are sufficiently strong to transport mass and to stretch the potential vorticity. A direct consequence is that such strongly stratified flows are not expected to be governed by two-dimensional horizontal Euler equations.

In this paper, we have focused on the most striking prediction of the asymptotic analysis. However, this is only a foretaste since our analysis provides extensive quantitative results, notably the dispersion relation and the spatial structure of the zigzag eigenmode, which deserves a complete test. To this end and to extend the present analysis to finite horizontal Froude number, wavenumber and Reynolds number, a numerical stability analysis is carried out in a companion paper (Billant \& Chomaz 2000b). For high Reynolds number, this investigation fully confirms both qualitatively and quantitatively the present asymptotic analysis while, for low Reynolds number, the numerical results agree with experimental measurements.

As a final remark, it may be noted that the present long-wavelength analysis could be carried out for arbitrary horizontal Froude number. However, the calculations would be far more difficult. Nonetheless, the problem in the limit $F_{h} \rightarrow \infty$ is tractable as shown in Appendix E. This analysis indicates that the zigzag instability does not occur in homogenous fluid in the long-wavelength limit. Therefore, the zigzag instability is likely to be peculiar to strongly stratified fluids $F_{h} \ll 1$ as indeed observed experimentally (Billant \& Chomaz 2000a).

The authors wish to thank L. S. Tuckerman for her fruitful comments and careful reading of the manuscript. This work has benefited from stimulating discussions with Olivier Thual and Stephan Fauve. 


\section{Appendix A. Adjoint $L^{\dagger}$}

The adjoint operator $L^{\dagger}$ is defined by

$$
\langle\tilde{\psi} \mid L(\psi)\rangle=\left\langle L^{\dagger}(\tilde{\psi}) \mid \psi\right\rangle
$$

where $L$ is defined in (3.23) and the scalar product is defined in (3.22). After some integrations by parts, the following is obtained:

$$
\begin{aligned}
\langle\tilde{\psi} \mid L(\psi)\rangle= & \int_{0}^{2 \pi} \int_{0}^{1} \psi\left[-\Delta\left(J\left(\psi_{0}, \tilde{\psi}\right)\right)+\mathscr{F}^{\prime}\left(\psi_{0}\right) J\left(\psi_{0}, \tilde{\psi}\right)\right] r \mathrm{~d} r \mathrm{~d} \theta \\
& -\int_{0}^{2 \pi}\left[r \frac{\partial \psi}{\partial r} J\left(\psi_{0}, \tilde{\psi}\right)-\psi r \frac{\partial J\left(\psi_{0}, \tilde{\psi}\right)}{\partial r}\right]_{0}^{1} \mathrm{~d} \theta \\
& -\int_{0}^{1}\left[\frac{1}{r} \frac{\partial \psi}{\partial \theta} J\left(\psi_{0}, \tilde{\psi}\right)-\frac{\psi}{r} \frac{\partial J\left(\psi_{0}, \tilde{\psi}\right)}{\partial \theta}\right]_{0}^{2 \pi} \mathrm{d} r .
\end{aligned}
$$

Therefore, the adjoint operator is

$$
L^{\dagger}(\tilde{\psi})=-\Delta\left(J\left(\psi_{0}, \tilde{\psi}\right)\right)+\mathscr{F}^{\prime}\left(\psi_{0}\right) J\left(\psi_{0}, \tilde{\psi}\right),
$$

and the adjoint boundary conditions will be chosen such that the boundary integrals disappear in (A 2).

To obtain these adjoint boundary conditions, we must first consider those for $\psi$. Clearly, $\psi$ and its derivatives must be $2 \pi$ periodic in $\theta$. Thus, $\tilde{\psi}$ is also chosen to be $2 \pi$ periodic in $\theta$. In order to derive the boundary condition at $r=1$ for $\psi$, the streamfunction $\psi$ is written as

$$
\psi=\sum_{m=0}^{\infty} \psi_{c}^{(m)}(r) \cos m \theta+\sum_{m=0}^{\infty} \psi_{s}^{(m)}(r) \sin m \theta
$$

For $r>1$, the perturbation streamfunction $\psi$ must be potential, $\Delta_{h} \psi=0$. The solution which remains bounded at $r=\infty$ is of the form

$$
\psi=\sum_{m=0}^{\infty} \frac{c_{m}}{r^{m}} \cos m \theta+\sum_{m=0}^{\infty} \frac{d_{m}}{r^{m}} \sin m \theta,
$$

in which solutions of the form $r^{m} \cos m \theta$ and $r^{m} \sin m \theta$ have been discarded because the associated perturbation velocity is unbounded at $r=\infty$. The solution $\psi=\ln r$ is not included because it is inconsistent with the requirement, derived by integrating (2.9a), that the circulation $\int_{0}^{2 \pi} \int_{0}^{1} \Delta_{h} \psi r \mathrm{~d} r \mathrm{~d} \theta$ of the perturbation at $r=1$ be zero at all times. Therefore, the boundary conditions (iii) and (iv) (see §2.4) impose

$$
m \psi_{c}^{(m)}(1)+\psi_{c}^{(m)^{\prime}}(1)=0,
$$

for $\psi_{c}^{(m)}$ for each $m$. The same boundary condition applies for $\psi_{s}^{(m)}$. To find the adjoint boundary condition at $r=1$, we also write $\tilde{\psi}=\sum_{m=0}^{\infty}\left(\tilde{\psi}_{c}^{(m)}(r) \cos m \theta+\tilde{\psi}_{s}^{(m)}(r) \sin m \theta\right)$. The adjoint boundary conditions for $\tilde{\psi}_{c}^{(m)}$ which make zero the first boundary integral in (A 2), are for $m \geqslant 2$

$$
(m-2)\left[(m-1) \tilde{\psi}_{c}^{(m-1)}(1)-(m+1) \tilde{\psi}_{c}^{(m+1)}(1)\right]+(m-2) \tilde{\psi}_{c}^{(m-1)^{\prime}}(1)-(m+2) \tilde{\psi}_{c}^{(m+1)^{\prime}}(1)=0,
$$


and

$$
\begin{gathered}
\tilde{\psi}_{c}^{(1)}(1)-\tilde{\psi}_{c}^{(1)^{\prime}}(1)=0, \\
2 \tilde{\psi}_{c}^{(2)}(1)-3 \tilde{\psi}_{c}^{(2)^{\prime}}(1)-2 \tilde{\psi}_{c}^{(0)^{\prime}}(1)=0 .
\end{gathered}
$$

The same boundary condition applies for $\tilde{\psi}_{s}^{(m)}$.

The boundary condition (ii) (see $\S 2.4$ ) at $r=0$ are that $\psi$ be non-singular. Accordingly, $\tilde{\psi}$ is also chosen to be non-singular at $r=0$.

\section{Appendix B. Determination of $\psi_{2}$}

\section{B.1. Solution of (3.36)}

Using (2.7a-b) and (3.18), the right-hand side $H_{2}$ of (3.36) for $r \leqslant 1$ can be easily written in the form

$$
H_{2}=H_{2}^{(1)}(r) \cos \theta+H_{2}^{(3)}(r) \cos 3 \theta+H_{2}^{(5)}(r) \cos 5 \theta,
$$

where

$$
\begin{gathered}
H_{2}^{(1)}=-\frac{\mu_{1}}{r J_{0}\left(\mu_{1}\right)}\left[2 \operatorname{Dr} J_{1}\left(\mu_{1} r\right)+\left(r J_{1}\left(\mu_{1} r\right) \chi_{2}^{(2)^{\prime}}\right)^{\prime}-2 \chi_{2}^{(2)} \frac{J_{1}\left(\mu_{1} r\right)}{r}\right], \\
H_{2}^{(3)}=-\frac{\mu_{1}}{r J_{0}\left(\mu_{1}\right)}\left[\left(r J_{1}\left(\mu_{1} r\right)\left(\chi_{2}^{(4)^{\prime}}-\chi_{2}^{(2)^{\prime}}\right)\right)^{\prime}+6\left(\chi_{2}^{(2)}-2 \chi_{2}^{(4)}\right) \frac{J_{1}\left(\mu_{1} r\right)}{r}\right], \\
H_{2}^{(5)}=-\frac{\mu_{1}}{r J_{0}\left(\mu_{1}\right)}\left[-\left(r J_{1}\left(\mu_{1} r\right) \chi_{2}^{(4)^{\prime}}\right)^{\prime}+20 \chi_{2}^{(4)} \frac{J_{1}\left(\mu_{1} r\right)}{r}\right] .
\end{gathered}
$$

Let $G$ be of the form

$$
G=G^{(0)}(r)+G^{(2)}(r) \cos 2 \theta+G^{(4)}(r) \cos 4 \theta .
$$

Then, equation (3.36) becomes a system of first-order differential equations

$$
\begin{gathered}
J_{1}\left(\mu_{1} r\right) G^{(4)^{\prime}}-4 \mu_{1} J_{1}^{\prime}\left(\mu_{1} r\right) G^{(4)}=r \mu_{1} J_{0}\left(\mu_{1}\right) H_{2}^{(5)}, \\
J_{1}\left(\mu_{1} r\right) G^{(2)^{\prime}}-2 \mu_{1} J_{1}^{\prime}\left(\mu_{1} r\right) G^{(2)}+J_{1}\left(\mu_{1} r\right) G^{(4)^{\prime}}+4 \mu_{1} J_{1}^{\prime}\left(\mu_{1} r\right) G^{(4)}=r \mu_{1} J_{0}\left(\mu_{1}\right) H_{2}^{(3)}, \\
2 J_{1}\left(\mu_{1} r\right) G^{(0)^{\prime}}+J_{1}\left(\mu_{1} r\right) G^{(2)^{\prime}}+2 \mu_{1} J_{1}^{\prime}\left(\mu_{1} r\right) G^{(2)}=r \mu_{1} J_{0}\left(\mu_{1}\right) H_{2}^{(1)},
\end{gathered}
$$

which is solved successively, e.g. $G^{(4)}$ is first determined, then $G^{(2)}$ and finally $G^{(0)}$. The numerical integration is started at the singular point $r=0$ by using asymptotic expression of the solutions.

\section{B.2. Determination of the function $\mathscr{K}\left(\psi_{0}\right)$}

In this appendix, we show that $\mathscr{K}\left(\psi_{0}\right)$ is determined on the basis of a solvability condition at order $F_{v}^{3}$. The equation at order $F_{v}^{3}$ for the streamfunction $\psi_{3}$ is (see (C 5))

$$
L\left(\psi_{3}\right)=-\left[\nabla_{h} \cdot\left(\Delta \psi_{0} \nabla_{h} \chi_{3}\right)+\Delta_{h} \Psi_{2}\right] \frac{\partial^{2} \phi_{1}}{\partial z^{2}}+\frac{\partial \Delta \psi_{0}}{\partial y} \frac{\partial \eta}{\partial T_{3}} \quad(r \leqslant 1) .
$$

The only solvability condition pertaining to this equation which is not automatically satisfied is

$$
\left\langle\left(\nabla_{h} \cdot\left(\Delta \psi_{0} \nabla_{h} \chi_{3}\right)+\Delta_{h} \Psi_{2}\right) \frac{\partial^{2} \phi_{1}}{\partial z^{2}}-\frac{\partial \Delta \psi_{0}}{\partial y} \frac{\partial \eta}{\partial T_{3}} \mid \mathscr{G}\left(\psi_{0}\right)\right\rangle=0,
$$


where $\mathscr{G}\left(\psi_{0}\right)$ stands for any function of $\psi_{0}$. This condition reduces to

$$
\left\langle\Delta_{h} \Psi_{2} \mid \mathscr{G}\left(\psi_{0}\right)\right\rangle=0,
$$

since the first and last terms in (B 8) integrate to zero while the vertical derivative of $\phi_{1}$ can be taken out of the scalar product. In order to obtain a more convenient expression for this constraint, we use streamline coordinates with orthogonal unit vectors $(\boldsymbol{T}, \boldsymbol{N})$, where $\boldsymbol{T}$ is tangent to the local direction of the basic velocity $\boldsymbol{u}_{h 0}$ and $N$ the principal normal to the streamline of the basic state. If $(s, n)$ represent coordinates measuring the distance in these two directions, respectively, we have

$$
\left\langle\Delta_{h} \Psi_{2} \mid \mathscr{G}\left(\psi_{0}\right)\right\rangle=\iint_{\mathscr{D}} \Delta_{h} \Psi_{2} \mathscr{G}\left(\psi_{0}\right) \mathrm{d} s \mathrm{~d} n,
$$

where $\mathscr{D}$ is the domain defined by $r \leqslant 1$. Instead of using the variable $n$, it is more convenient to use the streamfunction $\psi_{0}$ which is a function of $n$ only. The variations of $n$ and $\psi_{0}$ are simply related by $\partial \psi_{0} / \partial n=-u_{0}$ where $u_{0}=\sqrt{\boldsymbol{u}_{h 0} \cdot \boldsymbol{u}_{h 0}}$. Using now the variables $\left(s, \psi_{0}\right)$, the previous expression becomes

$$
\left\langle\Delta_{h} \Psi_{2} \mid \mathscr{G}\left(\psi_{0}\right)\right\rangle=-\int_{\psi_{0} \min }^{\psi_{0} \max }\left[\oint_{\gamma\left(\psi_{0}\right)} \frac{\Delta_{h} \Psi_{2}}{u_{0}} \mathrm{~d} s\right] \mathscr{G}\left(\psi_{0}\right) \mathrm{d} \psi_{0},
$$

where $\gamma\left(\psi_{0}\right)$ denotes a contour corresponding to a value of the streamfunction $\psi_{0}$ and $\psi_{0 \min }$ and $\psi_{0 \max }=-\psi_{0 \text { min }}$ are the minimum and maximum values of $\psi_{0}$ inside the circle $r=1$. Hence, a necessary and sufficient condition guaranteeing (B 8) for any function $\mathscr{G}\left(\psi_{0}\right)$ is

$$
\oint_{\gamma\left(\psi_{0}\right)} \frac{\Delta_{h} \Psi_{2}}{u_{0}} \mathrm{~d} s=0
$$

for each closed contour $\gamma$ corresponding to a value of the streamfunction $\psi_{0}$ inside the circle $r=1$. The solution of (3.37) for $r \leqslant 1$ can be formally written as $\Psi_{2}=M^{-1}(\mathscr{K}+G)$ which substituted in (B 11) gives a linear equation for $\mathscr{K}$

$$
\oint_{\gamma\left(\psi_{0}\right)} \frac{\Delta_{h} M^{-1}(\mathscr{K})}{u_{0}} \mathrm{~d} s=-\oint_{\gamma\left(\psi_{0}\right)} \frac{\Delta_{h} M^{-1}(G)}{u_{0}} \mathrm{~d} s,
$$

for $\psi_{0 \text { min }} \leqslant \psi_{0} \leqslant \psi_{0 \text { max }}$. This equation determines completely the function $\mathscr{K}\left(\psi_{0}\right)$ up to a constant. Without loss of generality, this constant is set to zero. The numerical procedure used to solve this equation is explained in Appendix B.3. When the streamlines are circular, the curvilinear coordinate $s$ is the azimuthal coordinate $s \equiv \theta$ and the solvability condition (B 11) is an average over $\theta$. This particular condition is often encountered in various asymptotic studies of vortices (see, for instance, Moffatt, Kida \& Ohkitani 1994). If $\mathscr{K}\left(\psi_{0}\right)$ satisfies (B 12), it is demonstrated in Appendix B.4 that a solution to (3.37) does exist. Once $\mathscr{K}\left(\psi_{0}\right)$ has been determined, the Helmholtz equation (3.37) can be solved numerically with a solution of the form (3.38). Note that since any function of the form $\partial \psi_{0} / \partial y$ may be added to the solution $\Psi_{2}$, the normalization condition

$$
\left\langle\Psi_{2} \mid \frac{\partial \psi_{0}}{\partial y}\right\rangle=0
$$

is further imposed. For $r>1$, the solution is potential and the Fourier components are of the form $\Psi_{2}^{(2 n)}(r)=b_{2 n} / r^{2 n}$ where the constants $b_{2 n}$ ensuring continuity at $r=1$ are $b_{0}=-0.6266, b_{2}=-1.3594, b_{4}=0.3248, \ldots$. 


\section{B.3. Numerical solution of (B 12)}

Details are given here for how to solve numerically the linear equation (B 12). The function $\mathscr{K}\left(\psi_{0}\right)$ is first discretized on $N$ points at $\psi_{0}=\psi_{0}^{j}, j=1, \ldots, N$ between 0 and $\psi_{0 \max }$. It is not necessary to consider the interval $\psi_{0 \text { min }} \leqslant \psi_{0} \leqslant 0$ because $\mathscr{K}\left(\psi_{0}\right)$ is an even function of $\psi_{0}$ as can be inferred from (B 12). Then, $\mathscr{K}$ is a vector $\mathscr{K}=\left[\mathscr{K}_{1}, \mathscr{K}_{2}, \ldots, \mathscr{K}_{N}\right]^{t}$, and (B 12) becomes a matrix-type relation

$$
A \mathscr{K}=-b
$$

where $A$ is the $N \times N$ matrix of the operator

$$
A=\oint_{\gamma\left(\psi_{0}\right)} \frac{\Delta_{h} M^{-1}}{u_{0}} \mathrm{~d} s
$$

and $b=\left[b_{1}, b_{2}, \ldots, b_{N}\right]^{t}$ is the right-hand side of (B 12) evaluated on every streamline $\psi_{0}^{j}$

$$
b_{j}=\oint_{\gamma\left(\psi_{0}^{j}\right)} \frac{\Delta_{h} M^{-1} G}{u_{0}} \mathrm{~d} s .
$$

The matrix $A$ is built numerically by taking successively for $i=1, \ldots, N$ a vector of the form

$$
k_{i}=\left[k_{i}^{1}, k_{i}^{2}, \ldots, k_{i}^{N}\right]^{t}
$$

where $k_{i}^{i}=1$ and $k_{i}^{j}=0$ for $j \neq i$. In other words, $k_{i}$ is zero everywhere except on the streamline $\psi_{0}^{i}$ where $k_{i}\left(\psi_{0}^{i}\right)=1$. Then, the product $A k_{i}$ is evaluated and yields column $i$ of the matrix $A$.

We now explain the procedure used to compute the product $A k_{i}$. We first solve the equation

$$
M\left(\psi_{i}\right)=k_{i},
$$

by expressing the function $k_{i}$ as a truncated Fourier series

$$
k_{i}=\sum_{n=0}^{N_{F}} a_{i}^{(2 n)}(r) \cos 2 n \theta,
$$

with $N_{F}+1$ modes. Only modes of the form $\cos 2 n \theta$ are relevant since $\mathscr{K}\left(\psi_{0}\right)$ is an even function of $\psi_{0}$. Accordingly, the solution $\psi_{i}$ is sought in the form $\psi_{i}=$ $\sum_{n=0}^{N_{F}} \psi_{i}^{(2 n)}(r) \cos 2 n \theta$. Then, (B 18) becomes a set of ordinary differential equations

$$
\frac{1}{r}\left(r \psi_{i}^{(2 n)^{\prime}}\right)^{\prime}+\psi_{i}^{(2 n)}\left(\mu_{1}^{2}-\frac{4 n^{2}}{r^{2}}\right)=a_{i}^{(2 n)}
$$

which are solved for $0 \leqslant n \leqslant N_{F}$. Finally, the integral is evaluated on every streamline $\psi_{0}^{j}$

$$
\oint_{\gamma\left(\psi_{0}^{j}\right)} \frac{\Delta_{h} \psi_{i}}{u_{0}} \mathrm{~d} s=A_{j i}
$$

for $j=1, \ldots, N$. Therefore, we have determined column $i$ of matrix $A$. The same procedure is applied to calculate $b_{j}$ for $j=1, \ldots, N$.

Then, equation (B 12) consists in solving the linear system

$$
A_{j i} \mathscr{K}_{i}=-b_{j},
$$

with a standard matrix linear algebra package. The number of points used is $N=60$ 
and the truncation order is $N_{F}=40$. Varying the truncation order and the number of points does not alter the results.

\section{B.4. Solvability conditions of (3.37)}

The solvability condition (3.26) does not ensure that (3.37) has a solution for any function $\mathscr{K}\left(\psi_{0}\right)$. However, we show in this appendix that if $\mathscr{K}\left(\psi_{0}\right)$ is chosen such that (B 11) is satisfied then a solution exists.

The Helmholtz operator $M$ defined in (3.37) with the associated boundary conditions (ii)-(iv) (see $\S 2.4$ ) is self-adjoint. The eigensolutions of $M$ for $r \leqslant 1$ are $J_{0}\left(\mu_{1} r\right)$ and $J_{2}\left(\mu_{1} r\right) \cos 2 \theta$ (or $J_{2}\left(\mu_{1} r\right) \sin 2 \theta$ ). This leads to two solvability conditions for the right-hand side of (3.37)

$$
\begin{gathered}
\left\langle G+\mathscr{K}\left(\psi_{0}\right) \mid J_{0}\left(\mu_{1} r\right)\right\rangle=0, \\
\left\langle G+\mathscr{K}\left(\psi_{0}\right) \mid J_{2}\left(\mu_{1} r\right) \cos 2 \theta\right\rangle=0 .
\end{gathered}
$$

However, the solvability condition (3.26) ensures only that

$$
\left\langle G+\mathscr{K}\left(\psi_{0}\right) \mid J_{0}\left(\mu_{1} r\right)+J_{2}\left(\mu_{1} r\right) \cos 2 \theta\right\rangle=0,
$$

which is obtained after integration by parts of (3.26) using the identity

$$
J_{0}\left(\mu_{1} r\right)+J_{2}\left(\mu_{1} r\right) \cos 2 \theta=J_{0}\left(\mu_{1}\right) J\left(\psi_{0}, r \cos \theta\right) .
$$

However, if $\mathscr{K}\left(\psi_{0}\right)$ is chosen such that condition (B 11) is satisfied, then we can write $\Delta \Psi_{2}=J\left(\psi_{0}, Q\right)$. On one hand, this implies that

$$
\left\langle\Delta_{h} \Psi_{2}\right\rangle=2 \pi \Psi_{2}^{(0)^{\prime}}(1)=0,
$$

because the Jacobian integrates to zero. On the other hand, taking the scalar product of (3.37) and $J_{0}\left(\mu_{1} r\right)$ gives

$$
\left\langle G+\mathscr{K}\left(\psi_{0}\right) \mid J_{0}\left(\mu_{1} r\right)\right\rangle=2 \pi J_{0}\left(\mu_{1}\right) \Psi_{2}^{(0)^{\prime}}(1) .
$$

Therefore, (B 27) and (B 28) imply that (B 23) is satisfied. Next, (B 25) implies that (B 24) is satisfied.

\section{Appendix C. Higher-order problems}

\section{C.1. Order $F_{v}^{3}$ problem}

In the two remaining orders to be solved $O\left(F_{v}^{3}\right)$ and $O\left(F_{v}^{4}\right)$, we are interested only in the equations for the horizontal motions. At these orders, the density and the vertical velocity do not need to be determined since they will only affect the horizontal motion at order $F_{v}^{5}$ and higher. At order $F_{v}^{3}$, the equations for the horizontal motions are

$$
\begin{aligned}
\boldsymbol{u}_{h 0} \cdot \nabla_{h} \tilde{\zeta}_{3}+\tilde{\boldsymbol{u}}_{h 3} \cdot \nabla_{h} \Delta \psi_{0} & =\Delta \psi_{0} \frac{\partial \tilde{u}_{z 1}}{\partial z}-\frac{\partial \tilde{\zeta}_{0}}{\partial T_{3}}-\frac{\partial \tilde{\zeta}_{2}}{\partial T_{1}}, \\
\nabla_{h} \cdot \tilde{\boldsymbol{u}}_{h 3}+\frac{\partial \tilde{u}_{z 1}}{\partial z} & =0 .
\end{aligned}
$$

The calculation follows the same procedure as used for the $F_{v}^{2}$ order. Writing the horizontal velocity with a potential $\chi_{3}(r, \theta)$ and a streamfunction $\psi_{3}\left(r, \theta, z, T_{1}, T_{3}\right)$ in the form

$$
\tilde{\boldsymbol{u}}_{h 3}=-\nabla \times\left(\psi_{3} \boldsymbol{e}_{z}\right)+\nabla_{h} \chi_{3} \frac{\partial^{2} \phi_{1}}{\partial z^{2}},
$$


and using expression (3.14) of the vertical velocity $\tilde{u}_{z 1}$, gives for the divergence equation $(\mathrm{C} 1 b)$

$$
\Delta_{h} \chi_{3}=-\frac{\partial P_{0}}{\partial y}+\nabla_{h} \cdot\left(\frac{\partial P_{0}}{\partial \theta} \boldsymbol{u}_{h 0}\right)=D_{3} .
$$

$D_{3}$ is written out in Appendix D. The solution is of the form

$$
\chi_{3}(r, \theta)=\chi_{3}^{(1)}(r) \sin \theta+\chi_{3}^{(3)}(r) \sin 3 \theta,
$$

where only $\chi_{3}^{(1)}(r)$ will be useful for expressing the next solvability condition. This function is shown in figure 5. Upon substituting the decomposition (C2) in the equation for the vertical vorticity $(\mathrm{C} 1 a)$ and by using (3.7b), (3.35) and (3.14), we obtain

$$
\begin{array}{ll}
L\left(\psi_{3}\right)=-\left[\nabla_{h} \cdot\left(\Delta \psi_{0} \nabla_{h} \chi_{3}\right)+\Delta_{h} \Psi_{2}\right] \frac{\partial^{2} \phi_{1}}{\partial z^{2}}+\frac{\partial \Delta \psi_{0}}{\partial y} \frac{\partial \eta}{\partial T_{3}} & (r \leqslant 1), \\
\Delta_{h} \psi_{3}=0 & (r>1) .
\end{array}
$$

The first term on the right-hand side of (C 5) has the same form as that at the previous order. In fact, a term of this form will appear systematically at all higher orders. The only solvability condition not automatically satisfied by the right-hand side of (C 5) is

$$
\left\langle\left(\nabla_{h} \cdot\left(\Delta \psi_{0} \nabla_{h} \chi_{3}\right)+\Delta_{h} \Psi_{2}\right) \frac{\partial^{2} \phi_{1}}{\partial z^{2}}-\frac{\partial \Delta \psi_{0}}{\partial y} \frac{\partial \eta}{\partial T_{3}} \mid \mathscr{G}\left(\psi_{0}\right)\right\rangle=0,
$$

where $\mathscr{G}\left(\psi_{0}\right)$ denotes any function of $\psi_{0}$. The first and last terms integrate to zero so that this solvability condition reduces to $\left\langle\Delta_{h} \Psi_{2} \mid \mathscr{G}\left(\psi_{0}\right)\right\rangle=0$. As explained in $\S 3.2$ and in Appendix B.2, the free function $\mathscr{K}\left(\psi_{0}\right)$ defined in (3.37) has been chosen so that $\Psi_{2}$ satisfies this condition.

Moreover, as for the first order, it is convenient to define a phase variable $\phi_{3}$ such that

$$
\frac{\partial \eta}{\partial T_{3}}=\phi_{3}
$$

The solution of (C 5) can be determined numerically in the form

$$
\psi_{3}=\Psi_{3} \frac{\partial^{3} \eta}{\partial^{2} z \partial T_{1}}+\left(\frac{\partial \psi_{0}}{\partial \theta}+r \cos \theta\right) \phi_{3},
$$

where

$$
\Psi_{3}(r, \theta)=\Psi_{3}^{(1)}(r) \cos \theta+\Psi_{3}^{(3)}(r) \cos 3 \theta+\cdots .
$$

We proceed in two stages as for $\psi_{2}$ by first looking for a solution $\Gamma$ of

$$
J\left(\psi_{0}, \Gamma\right)=-\left[\nabla_{h} \cdot\left(\Delta \psi_{0} \nabla_{h} \chi_{3}\right)+\Delta_{h} \Psi_{2}\right] \equiv H_{3} .
$$

$H_{3}$ is of the form

$$
H_{3}=H_{3}^{(0)}(r)+H_{3}^{(2)}(r) \cos 2 \theta+H_{3}^{(4)}(r) \cos 4 \theta+\cdots .
$$

Consequently, we seek $\Gamma$ in the form

$$
\Gamma=\Gamma^{(1)}(r) \cos \theta+\Gamma^{(3)}(r) \cos 3 \theta+\cdots .
$$

Then $(\mathrm{C} 10)$ becomes a system of first-order ordinary differential equations where the function $\Gamma^{(n)}$ can be determined successively for increasing $n . \Gamma^{(1)}$ is given by

$$
J_{1}\left(\mu_{1} r\right) \Gamma^{(1)^{\prime}}(r)+\mu_{1} J_{1}^{\prime}\left(\mu_{1} r\right) \Gamma^{(1)}(r)=r \mu_{1} J_{0}\left(\mu_{1}\right) H_{3}^{(0)},
$$


where $H_{3}^{(0)}$ is

$$
H_{3}^{(0)}=-\frac{\mu_{1}}{r J_{0}\left(\mu_{1}\right)}\left(r J_{1}\left(\mu_{1} r\right) \chi_{3}^{(1)^{\prime}}\right)^{\prime}-\frac{1}{r}\left(r \psi_{2}^{(0)^{\prime}}\right)^{\prime} .
$$

Then, (C 13) can be directly integrated to yield

$$
\Gamma^{(1)}(r)=-\mu_{1}^{2} r \chi_{3}^{(1)^{\prime}}-\frac{\mu_{1} J_{0}\left(\mu_{1}\right)}{J_{1}\left(\mu_{1} r\right)} r \psi_{2}^{(0)^{\prime}} .
$$

Note that the necessity of the solvability condition (B 11) which in particular imposes $\psi_{2}^{(0)^{\prime}}(1)=0$ appears here since otherwise the last term in $(\mathrm{C} 15)$ would be singular at $r=1$. The other terms of $\Gamma$ have not been determined since only the first function $\Psi_{3}^{(1)}$ is needed for computing the coefficients of the remaining phase equation. Then, we solve

$$
\begin{aligned}
\Delta_{h} \Psi_{3}+\mu_{1}^{2} \Psi_{3}=\Gamma \quad & (r \leqslant 1), \\
\Delta_{h} \Psi_{3}=0 & (r>1) .
\end{aligned}
$$

In this case, an arbitrary function of $\psi_{0}$ is not considered because no term of the form $\cos (2 n+1) \theta$ can be obtained from such a function. The function $\Psi_{3}^{(1)}$ is displayed on figure 6 .

\section{C.2. Order $F_{v}^{4}$ problem}

At order $F_{v}^{4}$, the equations for the horizontal motion are

$$
\begin{aligned}
\boldsymbol{u}_{h 0} \cdot \nabla_{h} \tilde{\zeta}_{4}+\tilde{\boldsymbol{u}}_{h 4} \cdot \nabla_{h} \Delta \psi_{0} & =\Delta \psi_{0} \frac{\partial \tilde{u}_{z 2}}{\partial z}-\frac{\partial \tilde{\zeta}_{1}}{\partial T_{3}}-\frac{\partial \tilde{\zeta}_{3}}{\partial T_{1}}, \\
\nabla_{h} \cdot \tilde{\boldsymbol{u}}_{h 4}+\frac{\partial \tilde{u}_{z 2}}{\partial z} & =0 .
\end{aligned}
$$

In view of the expression (3.41) of the vertical velocity $\tilde{u}_{z 2}$, the horizontal velocity is again separated into rotational and potential velocities in the form

$$
\tilde{\boldsymbol{u}}_{h 4}=-\nabla \times\left(\psi_{4} \boldsymbol{e}_{z}\right)+\nabla_{h} \chi_{44} \frac{\partial^{4} \eta}{\partial z^{4}}+\nabla_{h} \chi_{42} \delta^{2} \frac{\partial^{2} \eta}{\partial z^{2}}
$$

which, inserted in (C 17), gives the following equations for the potentials $\chi_{44}$ and $\chi_{42}$

$$
\begin{gathered}
\Delta_{h} \chi_{44}=\boldsymbol{u}_{h 0} \nabla_{h} \Pi_{2}+D \frac{\partial P_{0}}{\partial \theta} \equiv D_{44}, \\
\Delta_{h} \chi_{42}=\boldsymbol{u}_{h 0} \cdot \nabla_{h}\left[\boldsymbol{u}_{h 0} \cdot \nabla_{h}\left[\nabla_{h} \cdot\left(\frac{\partial P_{0}}{\partial y} \boldsymbol{u}_{h 0}\right)\right]\right] \equiv D_{42} .
\end{gathered}
$$

The functions $D_{44}$ and $D_{42}$ are written out in Appendix D. The solutions are of the form

$$
\begin{aligned}
& \chi_{44}(r, \theta)=\chi_{44}^{(2)}(r) \sin 2 \theta+\chi_{44}^{(4)}(r) \sin 4 \theta+\cdots, \\
& \chi_{42}(r, \theta)=\chi_{42}^{(2)}(r) \sin 2 \theta+\chi_{42}^{(4)}(r) \sin 4 \theta+\cdots,
\end{aligned}
$$

where only the first components $\chi_{44}^{(2)}$ and $\chi_{42}^{(2)}$ will be involved in the coefficients that remain to be calculated. These two functions are shown in figure 5 . 
An equation for the streamfunction $\psi_{4}$ is obtained by inserting the expression $(\mathrm{C} 18)$ into (C 17) and by using (3.12), (3.29), (C 8) and (3.11b)

$$
\begin{array}{rlr}
L\left(\psi_{4}\right)= & -\left[\nabla_{h} \cdot\left(\Delta \psi_{0} \nabla_{h} \chi_{44}\right)+D \Delta_{h} \Psi_{3}\right] \frac{\partial^{4} \eta}{\partial z^{4}} & \\
& -\nabla_{h} \cdot\left(\Delta \psi_{0} \nabla_{h} \chi_{42}\right) \delta^{2} \frac{\partial^{2} \eta}{\partial z^{2}}-\frac{\partial \Delta \psi_{0}}{\partial \theta}\left(\frac{\partial \phi_{1}}{\partial T_{3}}+\frac{\partial \phi_{3}}{\partial T_{1}}\right) & (r \leqslant 1), \\
\Delta_{h} \psi_{4}= & 0 & (r>1) .
\end{array}
$$

Only the solvability condition involving $\tilde{\psi}_{b}$ is not trivially satisfied. To fulfil this condition, we require that

$$
\frac{\partial \phi_{1}}{\partial T_{3}}+\frac{\partial \phi_{3}}{\partial T_{1}}=g_{1} \delta^{2} \frac{\partial^{2} \eta}{\partial z^{2}}+g_{2} \frac{\partial^{4} \eta}{\partial z^{4}}
$$

with

$$
\begin{gathered}
g_{1}=-\frac{\left\langle\tilde{\psi}_{b} \mid \nabla_{h} \cdot\left(\Delta \psi_{0} \nabla_{h} \chi_{42}\right)\right\rangle}{\left\langle\tilde{\psi}_{b} \mid \frac{\partial \Delta \psi_{0}}{\partial \theta}\right\rangle}, \\
g_{2}=-\frac{\left\langle\tilde{\psi}_{b} \mid \nabla_{h} \cdot\left(\Delta \psi_{0} \nabla_{h} \chi_{44}\right)+D \Delta_{h} \Psi_{3}\right\rangle}{\left\langle\tilde{\psi}_{b} \mid \frac{\partial \Delta \psi_{0}}{\partial \theta}\right\rangle} .
\end{gathered}
$$

After several integrations by parts, these coefficients are obtained from the functions $\chi_{42}^{(2)}$ and $\chi_{44}^{(2)}$, respectively,

$$
\begin{gathered}
g_{1}=-\frac{\mu_{1}^{2}}{2 J_{0}\left(\mu_{1}\right)} \int_{0}^{1} \chi_{42}^{(2)}(r) J_{2}\left(\mu_{1} r\right) r \mathrm{~d} r=-56.4 \\
g_{2}=-\frac{\mu_{1}^{2}}{2 J_{0}\left(\mu_{1}\right)} \int_{0}^{1} \chi_{44}^{(2)}(r) J_{2}\left(\mu_{1} r\right) r \mathrm{~d} r-D \Psi_{3}^{(1)}(1)=-16.1 .
\end{gathered}
$$

\section{Appendix D. Expression of the forcing terms appearing in the divergence equations (3.17), (C 3), (C 20), (C 19)}

Only the functions needed to compute the coefficients of the phase equations are explicitly written. $D_{2}$ reads

$$
D_{2}=D_{2}^{(2)}(r) \sin 2 \theta+D_{2}^{(4)}(r) \sin 4 \theta,
$$

where

$$
\left.\begin{array}{rr}
D_{2}^{(2)}=-\frac{1}{r J_{0}^{3}\left(\mu_{1}\right)}\left(2 J_{1}^{\prime}\left(\mu_{1} r\right)(2 b-a)-2 J_{1}\left(\mu_{1} r\right) \frac{a^{\prime}}{\mu_{1}}\right) & (r \leqslant 1), \\
D_{2}^{(2)}=-\frac{4}{r^{6}}\left(\frac{1}{r^{2}}-3\right) & (r>1),
\end{array}\right\}
$$


The functions $a$ and $b$ read

$$
a=J_{1}\left(\mu_{1} r\right) J_{2}^{\prime}\left(\mu_{1} r\right) / r, \quad b=2 J_{1}^{\prime}\left(\mu_{1} r\right) J_{2}\left(\mu_{1} r\right) / r .
$$

$D_{3}$ is of the form

$$
D_{3}=D_{3}^{(1)}(r) \sin \theta+D_{3}^{(3)}(r) \sin 3 \theta,
$$

where $D_{3}^{(1)}$ is given by

$$
\left.\begin{array}{ll}
D_{3}^{(1)}=\frac{1}{J_{0}\left(\mu_{1}\right)^{2}}(a+b)-\frac{4}{r J_{0}^{3}\left(\mu_{1}\right)}\left(J_{1}^{2}\left(\mu_{1} r\right) J_{1}^{\prime}\left(\mu_{1} r\right)+J_{2}^{2}\left(\mu_{1} r\right) J_{0}\left(\mu_{1} r\right)\right) & (r \leqslant 1), \\
D_{3}^{(1)}=-\frac{6}{r^{5}} & (r>1),
\end{array}\right\}
$$

$D_{42}$ is of the form

$$
D_{42}=D_{42}^{(2)}(r) \sin 2 \theta+D_{42}^{(4)}(r) \sin 4 \theta+\cdots,
$$

where $D_{42}^{(2)}$ is given by

$$
\left.\begin{array}{ll}
D_{42}^{(2)}=-\frac{1}{\mu_{1} r J_{0}\left(\mu_{1}\right)}\left[\mu_{1} J_{1}^{\prime}\left(\mu_{1} r\right)\left(C_{1}-3 C_{3}\right)-J_{1}\left(\mu_{1} r\right)\left(C_{1}^{\prime}+C_{3}^{\prime}\right)\right] & (r \leqslant 1), \\
D_{42}^{(2)}=\frac{8}{r^{8}}\left[\frac{23}{r^{6}}-\frac{84}{r^{4}}+\frac{108}{r^{2}}-30\right] & (r>1) .
\end{array}\right\}
$$

The functions $C_{1}$ and $C_{3}$ read

$$
\begin{aligned}
& C_{1}=-\frac{1}{\mu_{1} r J_{0}\left(\mu_{1}\right)}\left[2 \mu_{1} J_{1}^{\prime}\left(\mu_{1} r\right) D_{2}^{(2)}+J_{1}\left(\mu_{1} r\right) D_{2}^{(2)^{\prime}}\right], \\
& C_{3}=\frac{1}{\mu_{1} r J_{0}\left(\mu_{1}\right)}\left[\mu_{1} J_{1}^{\prime}\left(\mu_{1} r\right)\left(2 D_{2}^{(2)}-4 D_{2}^{(4)}\right)-J_{1}\left(\mu_{1} r\right)\left(D_{2}^{(2)^{\prime}}+D_{2}^{(4)^{\prime}}\right] .\right.
\end{aligned}
$$

$D_{44}$ is of the form

$$
D_{44}=D_{44}^{(2)}(r) \sin 2 \theta+D_{44}^{(4)}(r) \sin 4 \theta+\cdots .
$$

Note that the horizontal pressure gradient $\nabla_{h} \Pi_{2}$ appearing in $D_{44}$ (see (C 19)) is obtained from the horizontal momentum equation at order $F_{v}^{2}$

$$
\nabla_{h} \Pi_{2} \frac{\partial^{2} \eta}{\partial z^{2}}=-\tilde{\zeta}_{2} \boldsymbol{e}_{z} \times \boldsymbol{u}_{h 0}-\zeta_{0} \boldsymbol{e}_{z} \times \tilde{\boldsymbol{u}}_{h 2}-\nabla_{h}\left(\boldsymbol{u}_{h 0} \tilde{\boldsymbol{u}}_{h 2}\right)+D \frac{\partial^{2} \eta}{\partial z^{2}} \nabla \times\left[\left(\frac{\partial \psi_{0}}{\partial \theta}+r \cos \theta\right) \boldsymbol{e}_{z}\right]
$$


$D_{44}^{(2)}$ is given by

$$
\begin{aligned}
D_{44}^{(2)}= & \frac{2 J_{1}\left(\mu_{1} r\right)}{J_{0}^{2}\left(\mu_{1}\right)}\left[J_{1}\left(\mu_{1} r\right)\left(\frac{u_{2 \theta}^{(0)}}{r}-D-\frac{u_{2 \theta}^{(4)}}{2 r}\right)+\mu_{1} J_{1}^{\prime}\left(\mu_{1} r\right)\left(u_{2 r}^{(2)}-\frac{u_{2 r}^{(4)}}{2}\right)\right] \\
& -\frac{u_{0 \theta}}{2 r}\left(-u_{2 r}^{(2)} u_{0 r}-2 u_{2 \theta}^{(2)} u_{0 \theta}+u_{2 \theta}^{(0)} u_{0 \theta}-\frac{3}{2}\left(u_{2 r}^{(4)} u_{0 r}-u_{2 \theta}^{(4)} u_{0 \theta}\right)\right) \\
& -\frac{u_{0 r}}{2}\left(u_{2 r}^{(2)} u_{0 r}+u_{2 \theta}^{(0)} u_{0 \theta}+\frac{1}{2}\left(u_{2 r}^{(4)} u_{0 r}-u_{2 \theta}^{(4)} u_{0 \theta}\right)\right)^{\prime} \\
& +\frac{D J_{2}\left(\mu_{1} r\right)}{J_{0}\left(\mu_{1}\right)}\left(\frac{4 J_{0}\left(\mu_{1} r\right)}{J_{0}\left(\mu_{1}\right)}-1\right) \quad(r \leqslant 1), \\
D_{44}^{(2)}= & \frac{7}{2 r^{12}}-\frac{1}{2 r^{10}}\left(41-26 b_{4}+26 c_{4}\right)-\frac{1}{2 r^{8}}\left(53-8 b_{2}+12 b_{4}+8 c_{2}-12 c_{4}\right) \\
& +\frac{1}{r^{6}}\left(-7-12 b_{2}+5 b_{4}+12 c_{2}-5 c_{4}\right)-\frac{3}{r^{4}}-\frac{3 D}{r^{2}} \quad(r>1),
\end{aligned}
$$

where

$$
\begin{aligned}
& u_{2 r}^{(2)}=\frac{2}{r} \Psi_{2}^{(2)}+\chi_{2}^{(2)^{\prime}}, \quad u_{2 r}^{(4)}=\frac{4}{r} \Psi_{2}^{(4)}+\chi_{2}^{(4)^{\prime}}, \quad u_{0 r}=\frac{2 J_{1}\left(\mu_{1} r\right)}{r \mu_{1} J_{0}\left(\mu_{1}\right)} \\
& \left.u_{2 \theta}^{(0)}=\Psi_{2}^{(0)^{\prime}}, \quad u_{2 \theta}^{(2)}=\frac{2}{r} \chi_{2}^{(2)}+\Psi_{2}^{(2)^{\prime}}, \quad u_{2 \theta}^{(4)}=\frac{4}{r} \chi_{2}^{(4)}+\Psi_{2}^{(4)^{\prime}}, \quad u_{0 \theta}=-\frac{2 J_{1}^{\prime}\left(\mu_{1} r\right)}{J_{0}\left(\mu_{1}\right)} .\right\}
\end{aligned}
$$

The constants $b_{2}, b_{4}$ are defined in Appendix B.2 and $c_{2}=-2.016, c_{4}=-0.205$.

\section{Appendix E. Can the zigzag instability occur in homogeneous fluid?}

In this appendix, we carry out the beginning of the same long-wavelength analysis but in homogeneous fluid $F_{h}=\infty$ instead of $F_{h} \rightarrow 0$. In this case, the long-wavelength assumption is $L_{v} \gg L_{h}$ and the slow timescale $T_{1}$ is $T_{1}=L_{h} t / L_{v}$. At leading order in vertical wavenumber, we still have an equation of the form

$$
\frac{\partial^{2} \eta}{\partial T_{1}^{2}}=\tilde{D} \frac{\partial^{2} \eta}{\partial z^{2}}
$$

like (3.33). The coefficient $\tilde{D}$ can be computed easily. In this case, the vertical momentum equation (3.2c) at leading order is replaced by

$$
\boldsymbol{u}_{h 0} \cdot \nabla_{h} \tilde{u}_{z 0}=\frac{\partial P_{0}}{\partial y} \frac{\partial \eta}{\partial z}
$$

which is solved easily for the vertical velocity

$$
\tilde{u}_{z 0}=-u_{y 0} \frac{\partial \eta}{\partial z}
$$

Thus, the divergence equation (3.17) is replaced by

$$
\Delta_{h} \chi_{2}=u_{y 0}
$$


Again, the solution for $r \leqslant 1$ is readily found

$$
\chi_{2}=\left(\frac{r^{2}}{8}-\frac{J_{2}\left(\mu_{1} r\right)}{\mu_{1}^{2} J_{0}\left(\mu_{1}\right)}\right) \sin 2 \theta,
$$

Substituting this expression into the relation (3.31), which remains valid for $F_{h}=\infty$, yields the coefficient

$$
\tilde{D}=-\frac{1}{2 \pi}\left\langle\Delta \psi_{0} \mid \frac{\partial \chi_{2}}{\partial x}\right\rangle=\frac{1}{2}
$$

Because $\tilde{D}$ is positive, the zigzag instability does not exist in the long-wavelength limit in homogeneous fluid.

\section{REFERENCES}

Batchelor, G. K. 1967 An Introduction to Fluid Dynamics. Cambridge University Press.

Billant, P. \& Chomaz, J.-M. $2000 a$ Experimental evidence for a new instability of a vertical columnar vortex pair in a strongly stratified fluid. J. Fluid Mech. 418, 167-188.

Billant, P. \& Chomaz, J.-M. $2000 b$ Three-dimensional stability of a vertical columnar vortex pair in a stratified fluid. J. Fluid Mech. 419, 65-91.

Bonnier, M., Eiff, O. \& Bonneton, P. 2000 On the density structure of far wake vortices in a stratified fluid. Dyn. Atmos. Oceans 31, 117-137.

Browand, F. K., Guyomar, D. \& Yoon, S.-C. 1987 The behavior of a turbulent front in a stratified fluid: Experiments with an oscillating grid. J. Geophys. Res. 92, 5329-5341.

Chomaz, J. M., Bonneton, P., Butet, A. \& Hopfinger, E. J. 1993 Vertical diffusion in the far wake of a sphere moving in a stratified fluid. Phys. Fluids A 5, 2799-2806.

Coullet, P. \& Fauve, S. 1985 Propagative phase dynamics for systems with Galilean invariance. Phys. Rev. Lett. 55, 2857-2859.

Cross, M. C. 1983 Phase dynamics of convective rolls. Phys. Rev. A 27, 490-498.

Cross, M. C. \& Newell A. C. 1984 Convection patterns in large aspect ratio systems. Physica 10D, 299-328.

Dritschel, D. G. \& Torre JuÁrez, M. DE LA 1996 The instability and breakdown of tall columnar vortices in a quasi-geostrophic fluid. J. Fluid Mech. 328, 129-160.

FAUVE, S. 1987 Large scale instabilities of cellular flows. In Instabilities and Nonequilibrium Structures (ed. E. Tirapegui \& D. Villarroel), pp. 63-88.

Fauve, S., Bolton, E. W. \& Brachet, M. E. 1987 Nonlinear oscillatory convection: a quantitative phase dynamics approach. Physica 29D, 202-214.

Fincham, A. M., Maxworthy, T. \& Spedding G. R. 1996 Energy dissipation and vortex structure in freely decaying stratified grid turbulence. Dyn. Atmos. Oceans 23, 171-182.

Flór, J. B. \& Heisst, G. J. F. van 1996 Stable and unstable monopolar vortices in a stratified fluid. J. Fluid Mech. 211, 257-287.

GreGG, M. C. 1987 Diapycnal mixing in the thermocline: a review. J. Geophys. Res. 92, 5249-5286.

Herring, J. R. \& MÉtais, O. 1989 Numerical simulations in forced stably stratified turbulence. J. Fluid Mech. 202, 97-115.

Hopfinger, E. J. 1987 Turbulence in stratified fluids: a review. J. Geophys. Res. 92, 5287-5303.

Itsweire, E. C., Helland, K. N. \& Van Atta, C. W. 1986 The evolution of grid-generated turbulence in a stably stratified fluid. J. Fluid Mech. 162, 299-338.

Kimura, Y. \& Herring, J. R. 1996 Diffusion in stably stratified turbulence. J. Fluid Mech. 328, 253-269.

Kuramoto, Y. 1984 Phase dynamics of weakly unstable periodic structures. Prog. Theor. Phys. 71, $1182-1197$.

Lamb, H. 1932 Hydrodynamics. Cambridge University Press.

LiLLY, D. K. 1983 Stratified turbulence and the mesoscale variability of the atmosphere. J. Atmos. Sci. 40, 749-761.

Lilly, D. K., Bassett, G., Droegemeier, K. \& Bartello, P. Stratified turbulence in the atmospheric mesoscales. Theor. Comput. Fluid Dyn. 11, 139-153. 
Lin, Q., Lindberg, W. R., Boyer, D. L. \& Fernando, H. J. S. 1992 Stratified flow past a sphere. J. Fluid Mech. 240, 315-354.

Lin, J. T. \& PAO, Y. H. 1979 Wakes in stratified fluids: a review. Ann. Rev. Fluid Mech. 11, 317-338.

MaJdA, A. J. \& Grote, M. J. 1998 Model dynamics and vertical collapse in decaying strongly stratified flows. Phys. Fluids 9, 2932-2940.

Manneville, P. 1990 Dissipative Structures and Weak Turbulence. Academic Press, Boston.

MeleshKo, V. V. \& HeiJst, G. J. F. van 1994 On Chaplygin's investigations of two-dimensional vortex structures in an inviscid fluid. J. Fluid Mech. 272, 157-182.

MÉtais, O. \& Herring, J. R. 1989 Numerical simulations of freely evolving turbulence in stably stratified fluids. J. Fluid Mech. 202, 117-148.

Moffatt, H. K., Kida, S. \& OhKitani, K. 1994 Stretched vortices - the sinews of turbulence; large-Reynolds-number asymptotics. J. Fluid Mech. 259, 241-264.

Pomeau, Y. \& Manneville, P. 1979 Stability and fluctuations of a spatially periodic flow. J. Physique Lettres 40, 609-612.

Riley, J. J., Metcalfe, R. W. \& Weissman, M. A. 1981 Direct numerical simulations of homogeneous turbulence in density stratified fluids. In Proc. AIP Conf. Nonlinear Properties of Internal Waves (ed. B. J. West), pp. 79-112.

Sipp, D., Jacquin, L. \& Cossu, C. 2000 Self-adaptation and viscous selection in concentrated two-dimensional vortex dipoles. Phys. Fluids 12, 245-248.

SpedDing, G. R. 1997 The evolution of initially turbulent bluff-body wakes at high internal Froude number. J. Fluid Mech. 337, 283-301.

Spedding, G. R., Browand, F. K. \& Fincham, A. M. 1996 Turbulence, similarity scaling and vortex geometry in the wake of a sphere in a stably-stratified fluid. J. Fluid Mech. 314, 53-103.

Staquet, C. \& Riley, J. J. 1989 On the velocity field associated with potential vorticity. Dyn. Atmos. Oceans 14, 93-123.

Stillinger, D. C., Helland, K. N. \& Van Atta, C. W. 1983 Experiments on the transition of homogeneous turbulence to internal waves in a stratified fluid. J. Fluid Mech. 131, 91-122.

Williamson, C. H. K. \& Chomaz, J. M. 1997 The exploding vortex pair. Phys. Fluids 9, 54. 